\section{RMD Open}

Rheumatic \&

Musculoskeletal Diseases

\title{
Accuracy of musculoskeletal imaging for the diagnosis of polymyalgia rheumatica: systematic review
}

Sarah Louise Mackie, ${ }^{1,2}$ Gouri Koduri, ${ }^{3,4}$ Catherine L Hill, ${ }^{5}$ Richard J Wakefield, ${ }^{1,2}$ Andrew Hutchings, ${ }^{6}$ Clement Loy, ${ }^{7,8}$ Bhaskar Dasgupta, ${ }^{9}$ Jeremy C Wyatt $^{10}$

To cite: Mackie SL, Koduri G, Hill $\mathrm{CL}$, et al. Accuracy of musculoskeletal imaging for the diagnosis of polymyalgia rheumatica: systematic review. $R M D$ Open 2015;1: e000100. doi:10.1136/ rmdopen-2015-000100

- Prepublication history and additional material is available. To view please visit the journal (http://dx.doi.org/ 10.1136/rmdopen-2015000100).

An earlier version of this work was presented in poster form at the American College of Rheumatology Annual Meeting in November, 2014.

Received 20 March 2015 Revised 18 May 2015 Accepted 31 May 2015

CrossMark

For numbered affiliations see end of article.

Correspondence to Dr Sarah Louise Mackie; s.l.mackie@leeds.ac.uk

\section{ABSTRACT}

Objectives: To review the evidence for accuracy of imaging for diagnosis of polymyalgia rheumatica (PMR).

Methods: Searches included MEDLINE, EMBASE and PubMed. Evaluations of diagnostic accuracy of imaging tests for PMR were eligible, excluding reports with $<10$ PMR cases. Two authors independently extracted study data and three authors assessed methodological quality using modified QUADAS-2 criteria.

Results: 26 studies of 2370 patients were evaluated: 10 ultrasound scanning studies; 6 MRI studies; 1 USS and MRI study; 7 18-fluorodeoxyglucose-positron emission tomography (PET) studies; 1 plain radiography and 1 technetium scintigraphy study. In four ultrasound studies, subacromial-subdeltoid bursitis had sensitivity $80 \%$ (95\% Cl 55\% to $93 \%)$ and specificity $68 \%(95 \% \mathrm{Cl} 60 \%$ to $75 \%)$, whereas bilateral subacromial-subdeltoid bursitis had sensitivity $66 \%(95 \% \mathrm{Cl} 43 \%$ to $87 \%)$ and specificity $89 \%$ (95\% $\mathrm{Cl} 66 \%$ to $97 \%$ ). Sensitivity for ultrasound detection of trochanteric bursitis ranged from $21 \%$ to $100 \%$. In four ultrasound studies reporting both subacromialsubdeltoid bursitis and glenohumeral synovitis, detection of subacromial-subdeltoid bursitis was more accurate than that of glenohumeral synovitis $(p=0.004)$. MRI and PET/CT revealed additional areas of inflammation in the spine and pelvis, including focal areas between the vertebrae and anterior to the hip joint, but the number of controls with inflammatory disease was inadequate for precise specificity estimates.

Conclusions: Subacromial-subdeltoid bursitis appears to be the most helpful ultrasound feature for PMR diagnosis, but interpretation is limited by study heterogeneity and methodological issues, including variability in blinding and potential bias due to casecontrol study designs. Recent MRI and PET/CT casecontrol studies, with blinded readers, yielded promising data requiring validation within a diagnostic cohort study.

Polymyalgia rheumatica (PMR) is an ageassociated, inflammatory musculoskeletal disease with a lifetime risk of $2.4 \%$ for women

Key messages
What is already known about this subject?
Provisional American College of Rheumatology/
the European League Against Rheumatism
(ACR/EULAR) classification criteria for polymyal-
gia rheumatica (PMR) incorporate several
optional ultrasound features, with intra-articular
and extra-articular features of inflammation
weighted equally.
What does this study add?
Subacromial-subdeltoid bursitis is significantly
more discriminatory for PMR compared to gle-
nohumeral synovitis, in four studies with ultra-
sound data on both features.
Data mostly come from diagnostic case-control
study designs, which can overestimate values
for sensitivity and specificity.
How might this impact on clinical practice?
When evaluating patients with suspected PMR,
clinicians may consider extra-articular locations
of inflammation such as bursitis as supportive,
but must bear in mind that there may be biases
in current estimates of sensitivity and specificity
of these findings.

and $1.7 \%$ for men, ${ }^{1}$ and affects $0.7 \%$ of the population over the age of 50 years. ${ }^{2}$ Patients report pain and stiffness of the shoulder and/ or hip girdles, usually with elevation of inflammatory markers such as $\mathrm{C}$ reactive protein and erythrocyte sedimentation rate. ${ }^{3}$ Accurate diagnosis of PMR is essential, given the impact of PMR on quality of life unless it is treated with systemic glucocorticoids, usually for a year or more. ${ }^{4}$ Long-term glucocorticoids produce a significant risk of adverse events. ${ }^{5-8}$ However, PMR can be mimicked by many other conditions, ${ }^{9}$ many of which also respond initially to glucocorticoids. None of the various sets of classification 
criteria for PMR has yet been fully validated for clinical diagnostic use. There remains a need for additional tests providing diagnostic information, especially where the diagnosis is not clear-cut.

In PMR, there is inflammation in and around the shoulders and hips; ${ }^{3}$ this can often be visualised using imaging. ${ }^{10}$ Based on small, single-centre studies, it has been hypothesised that PMR compared to RA has predominantly extra-articular rather than intra-articular imaging abnormalities. ${ }^{11-14}$ However, the latest, datadriven provisional international classification criteria for PMR give equal weighting to extra-articular and intra-articular ultrasound features. ${ }^{15}$ Since extra-articular features such as subacromial-subdeltoid bursitis (SAB) and trochanteric bursitis are commonly seen with normal ageing, ${ }^{16} 17$ it is important to compare any imaging findings with those from non-PMR controls of similar ages.

The objective of this study was to review the evidence regarding the accuracy of musculoskeletal imaging for the diagnosis of PMR.

\section{METHODS}

\section{Data sources and searches}

The systematic review protocol was uploaded to the PROSPERO database before running searches (registration number CRD42013005734). The reference standard was defined as a rheumatologist's diagnosis of PMR, without any better explanation of the presenting symptoms found during follow-up. Potential sources of heterogeneity, including study setting, eligibility criteria, technical aspects of the imaging and glucocorticoid therapy were pre-defined. A PICO-structured search was conducted to identify relevant studies in Pubmed, Ovid MEDLINE (1966-) and EMBASE (including EMBASE Classic) (table 1).

\section{Study selection}

A study was eligible if it included humans with either suspected PMR (diagnostic cohort design), or both a PMR group and a comparator non-PMR group (diagnostic case-control design), with systematic application of imaging test(s). Expert (rheumatologist) diagnosis was the minimum acceptable reference standard. Diagnostic accuracy data had to be extractable in $2 \times 2$ format (true positives, true negatives, false positives, false negatives). Non-systematic review articles, case reports and case series of less than 10 patients were excluded. No language restrictions were made. Case reports were excluded by the reviewers manually, rather than by using filters. Meeting abstracts (previous 2 years of British Society for Rheumatology (BSR), European League against Rheumatism (EULAR) and American College of Rheumatology (ACR) conferences) were also screened, and experts in the field were contacted, to identify studies potentially in press or not fully published. Citations were exported to EndNote, duplicates removed in EndNote and results exported to Microsoft Excel.
Table 1 Search strategy

Polymyalg* AND (imaging OR ultrasono* OR sonograph* OR echogr* OR "computed tomography" OR "computer assisted tomography" OR "bone scan" OR "nuclear medicine" OR "scintigraph"” OR "PET" OR

Pubmed: "positron" OR "MRl" OR "magnetic")

\section{Ovid Medline:}

1 Polymyalgia Rheumatica/

2 polymyalgi\$.mp

$3 \quad$ PMR.tw

$4 \quad$ exp Rheumatic Diseases/

$5 \quad 3$ and 4

$6 \quad 1$ or 2 or 5

7 human/

8 (editorial or comment or historical article or review).pt

$9 \quad 7$ not 8

10 exp "diagnostic imaging"/

11 (diagnostic imaging).mp

12 ri.fs

13 ra.fs

14 us.fs

15 mri.mp

16

17

18

19

20

21

22

23

24

25

26

27

28

29

30

31

32

33

34

Ovid EMBASE:

(magnetic resonance).mp

(mr imaging).mp

$\mathrm{mr}$ scan\$

mr.ti

exp ultrasonography/

ultrasound.mp

ultrason $\$ . m p$

echograph\$.mp

sonograph\$.mp

doppler\$.mp

us.ti

scintigraph\$.mp

positron.mp

PET.ti

ct.ti

radiograph $\$ . m p$

$\mathrm{x}$-ray $\$ \mathrm{mp}$

or/10-32

6 and 9 and 33

1 exp rheumatic polymyalgia/

2 polymyalgi\$.mp

$3 \quad$ PMR.tw

$4 \quad$ exp rheumatic disease/

$5 \quad 3$ and 4

$6 \quad 1$ or 2 or 5

$7 \quad$ limit 6 to human

$8 \quad$ limit 6 to editorial

$9 \quad$ limit 6 to review

107 not (8 or 9$)$

11 diagnostic imaging.mp.

12 exp diagnostic imaging/

13 radiodiagnosis/

14 exp echography/

15 exp computer assisted tomography/

16 exp nuclear magnetic resonance imaging/

Continued 
Table 1 Continued

\begin{tabular}{|c|c|}
\hline Pubmed: & $\begin{array}{l}\text { Polymyalg* AND (imaging OR ultrasono* } \\
\text { OR sonograph* OR echogr* OR "computed } \\
\text { tomography" OR “computer assisted } \\
\text { tomography" OR "bone scan" OR "nuclear } \\
\text { medicine" OR "scintigraph"” OR "PET" OR } \\
\text { "positron" OR “MRI" OR "magnetic") }\end{array}$ \\
\hline 17 & exp positron emission tomography/ \\
\hline 18 & ct.ti \\
\hline 19 & (mr imaging).mp \\
\hline 20 & (magnetic resonance).mp \\
\hline 21 & mri.mp \\
\hline 22 & mr.ti \\
\hline 23 & pet.mp \\
\hline 24 & positron.mp \\
\hline 25 & scintigraph\$.mp \\
\hline 26 & sonograph\$.mp \\
\hline 27 & ultraso\$.mp \\
\hline 28 & echograph\$.mp \\
\hline 29 & doppler\$.mp \\
\hline 30 & us.ti \\
\hline 31 & exp ultrasound \\
\hline 32 & di.fs \\
\hline 33 & radiograph\$.mp \\
\hline 34 & $\mathrm{x}$-ray\$.mp \\
\hline 35 & or/11-34 \\
\hline 36 & 10 and 35 \\
\hline
\end{tabular}

The search was performed by combining the following search terms: polymyalgia/polymyalgic and (ultrasound or radiograph or X-ray or imaging or CT or MRI or PET or CT or isotope bone scan or positron emission tomography or MR). No language restrictions were made, in case the abstract reveals useful information.

\section{Data extraction and quality assessment}

A study quality assessment tool, based on QUADAS-2, ${ }^{18}$ and encompassing internal validity (risk of bias: test reliability, blinding to index test/clinical information, incorporation bias, diagnostic review bias) and external validity (relevance to our review question: participant selection, spectrum of disease and comparator condition, timing of test in relation to glucocorticoid treatment) was agreed in advance. Two reviewers (SLM and GK) independently extracted study characteristics (design, clinical spectrum, reference standard) and diagnostic accuracy data for the index test(s) of each study. Corresponding authors were contacted by email where queries arose. Assessment of methodological limitations and between-study clinical heterogeneity was guided by the study quality assessment tool. Data were entered into Review Manager V.5.2 (RevMan) and exported to Excel.

\section{Data synthesis and analysis}

For each imaging feature, where 4 or more studies were available, meta-analysis was performed in Stata SE V.12 (StataCorp, Texas, USA) with calculation of overall sensitivity, specificity and likelihood ratios (LRs) using the bivariate model, ${ }^{19}$ and graphed using RevMan, allowing visualisation of between-study statistical heterogeneity.
Influential studies were identified by plotting Cook's distance for each study. Where fewer than four studies were available, $95 \%$ CIs for sensitivity, specificity and LRs for each study were calculated using a spreadsheet. ${ }^{20}$ If a cell in the $2 \times 2$ table for a study contained a $0,0.5$ was added to each cell to avoid division-by-zero.

To directly compare accuracy of specific couples of tests, we used Hierarchial Summary Receiver-Operator Characteristic Curve (HsROG) modelling, ${ }^{21}$ with test type as a covariate. We did so with paired data only (data from studies where both tests were evaluated together), to control for study-based biases. We first assessed whether the sROC's for the two tests had similar shapes (the beta parameter), as sROC's with different shapes will cross and whether one test is better than the other or not becomes threshold-dependent. ${ }^{22}$ Where the two sROC's had similar shapes, we were able to compare overall accuracy using the $\alpha$ parameter (indicating proximity to the top left hand corner of the ROC space). Analysis was performed using PROC NLMIXED in SAS V.9.3 (SAS Institute, North Carolina, USA).

\section{RESULTS}

Literature searches were completed on 2 October 2013, yielding 1764 citations (figure 1). We identified 87 articles for full text review of which 23 studies from the original searches were chosen for full evaluation, with three further added on updating searches (January 2015): 10 ultrasound scanning studies (including one published in full text on the updated $\operatorname{search}^{23}$ ); 6 MRI studies; 1 USS and MRI study; 7 18-fluorodeoxyglucose-positron emission tomography (PET) studies (including two published in full text when the search was updated ${ }^{24}$ ); 1 plain radiography $^{26}$ and 1 technetium scintigraphy ${ }^{27}$ study. These last two studies did not meet our review inclusion criteria, one because of a lack of clear distinction between PMR and non-PMR ${ }^{26}$ and the other because it was published in 1976 and we could not exclude the possibility that changes in definition of the diagnostic reference standard may have occurred since then. ${ }^{27}$ Additionally, we reviewed four longitudinal studies. ${ }^{28-31}$ Vascular imaging studies in patients with a diagnosis of PMR were also initially reviewed (six ultrasound and two PET), but subsequently excluded as the primary purpose of these studies was to diagnose giant cell arteritis in patients presenting with PMR symptoms.

Study characteristics and results of quality assessment are shown in table 2. All but one of the studies we identified used a diagnostic case-control design, which is associated with inflation of sensitivity and specificity estimates because of the 'grey cases' seen in real-life clinical practice but omitted from the study. ${ }^{32}$ Other common sources of bias in this analysis included incomplete blinding of the person(s) performing the imaging test, diagnostic review bias (incomplete blinding of the diagnostician acting as reference standard) and spectrum bias (studies were generally conducted in academic 


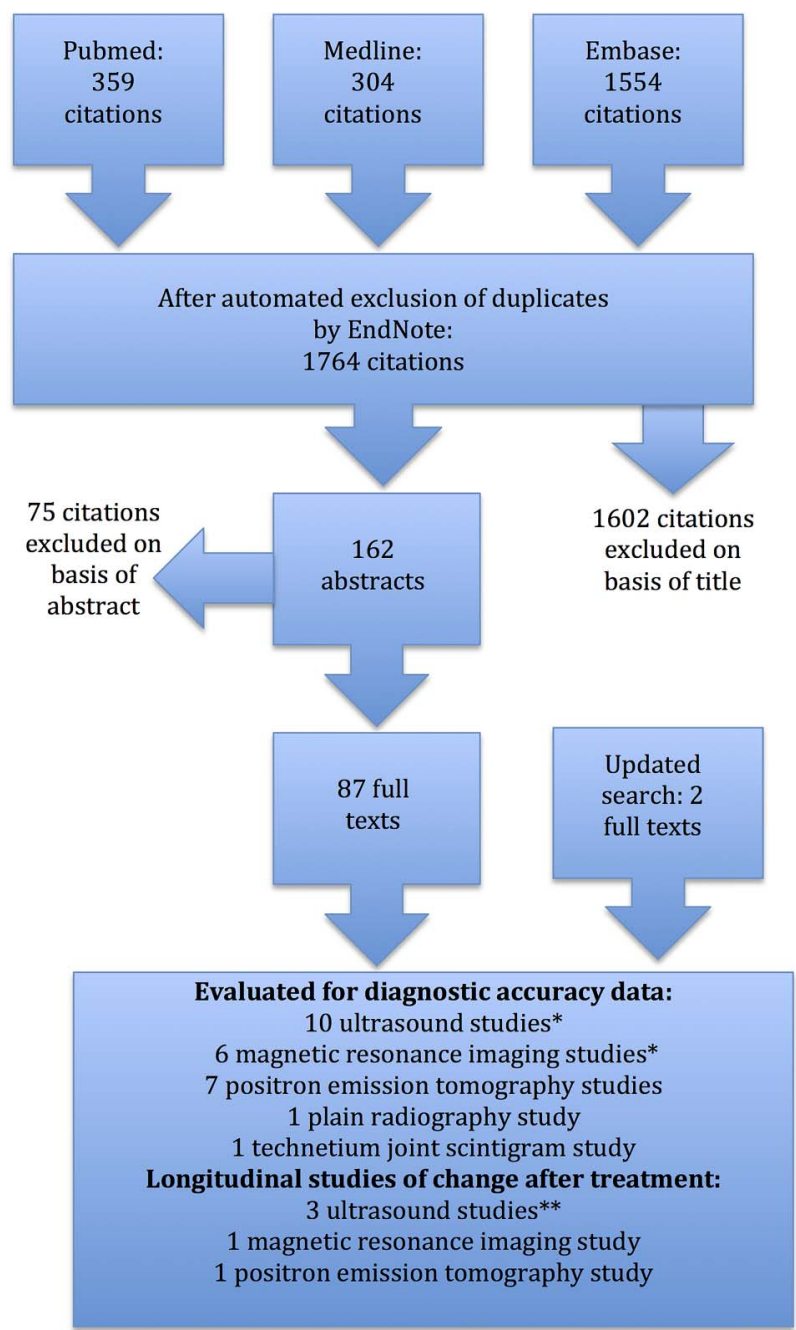

Figure 1 Flow chart for systematic review.

rheumatology centres) (table 2). Table 3 summarises the accuracy of each imaging feature in PMR, using meta-analysis where appropriate. Original data used to create this table and further details regarding comparator subpopulations are found in the online supplementary. Many different abnormalities were reported by the studies, reflecting the widespread localisation of inflammation in PMR.

\section{Accuracy of bursitis imaging (extracapsular inflammation)}

Meta-analysis of four USS studies gave a sensitivity of $80 \%(95 \%$ CI $55 \%$ to $93 \%)$ and specificity of $68 \%(60 \%$ to $75 \%$ ) for SAB; the same studies showed a sensitivity of $66 \%(36 \%$ to $87 \%)$ and specificity $89 \%$ (66\% to $97 \%$ ) for bilateral SAB. Examination of the HsROC plot indicates substantial heterogeneity of discrimination, with an early study ${ }^{33}$ showing much higher diagnostic accuracy than subsequent studies (figure 2).

Data on trochanteric bursitis were variable; very high sensitivity of ultrasound in an early single-centre study ${ }^{34}$ was not replicated in a later multicentre study. ${ }^{15}$ Pelvic-girdle symptoms were required for inclusion in the earlier study, whereas the later study required shoulder symptoms but did not require pelvic-girdle symptoms.

Other bursal sites around the hip/pelvic region (ischiogluteal, iliopsoas), while reportedly more specific for PMR than trochanteric bursitis, are technically difficult to detect using ultrasound compared to MRI. ${ }^{34}$ Although a PET/CT study suggested inflammation around the ischial tuberosity may be informative for PMR diagnosis, the sample size was small, and thus CIs for sensitivity and specificity are wide $;{ }^{35}$ sensitivity on an earlier MRI study was only $25 \% .{ }^{34}$ Similarly, inflammation (bursitis) between posterior vertebral elements, detectable by PET/CT ${ }^{25}{ }^{35}$ or MRI, ${ }^{36}{ }^{37}$ appeared to be highly specific compared to age-matched controls without inflammatory rheumatic disease, but may also be observed in $\mathrm{RA}^{25}$ most of the RA comparator patients were taking prednisolone (D Camellino, personal communication, January 2015). PET/CT can also identify iliopsoas (iliopectineal) bursitis, sometimes seen in RA as well. ${ }^{24}$

\section{Accuracy of imaging intracapsular inflammation and fluid around long head of biceps tendon}

Synovitis at shoulder (glenohumeral) or hip (coxofemoral) joints, and fluid around the long head of biceps tendon (which is related to synovial inflammation, since this space is synovium-lined and also communicates with the glenohumeral joint itself), were reported by several studies. Combining the ultrasound studies, glenohumeral synovitis had a sensitivity of $62 \%$ (95\% CI $46 \%$ to $76 \%$ ) and specificity of $58 \%$ (45\% to $69 \%$ ), and hip synovitis had a sensitivity of $33 \%$ (24\% to $43 \%)$ and specificity of $78 \%$ (66\% to $87 \%)$. MRI and PET/CT were much more sensitive for detecting hip synovitis in PMR, but with a loss of specificity.

\section{Comparison with RA}

Comparison with RA may identify imaging features specific to PMR and not seen in other inflammatory joint diseases. Two ultrasound studies recruited only patients with RA as controls. In both studies, to minimise the risk of misclassification, cases and controls were selected on the basis of already having an established diagnosis of (treated) PMR or RA. Methodological quality was difficult to assess in the earlier study, ${ }^{38}$ but the later study ${ }^{39}$ recruited only relapsing patients with new-onset bilateral shoulder pain; the authors reported in correspondence with us that low-dose prednisolone treatment did not seem to affect the ultrasound findings. It is difficult to recruit large numbers of patients with untreated RA and elevated inflammatory markers. One PET/CT study recruited 10 untreated RA patients ${ }^{24}$ but in another, the RA patients were on treatment. ${ }^{40}$

Combined features (defined by the provisional ACR/EULAR classification criteria for PMR

In the provisional ACR/EULAR classification criteria for PMR, ${ }^{15}$ ultrasound features of inflammation were 
Table 2 Assessment of methodological quality in diagnostic studies: summary of major biases identified

\begin{tabular}{|c|c|c|c|c|c|c|c|c|c|c|c|}
\hline Study & $\begin{array}{l}\text { Index test: } \\
\text { imaging } \\
\text { modality }\end{array}$ & $\begin{array}{l}\text { Who performed index test, } \\
\text { were they blinded to clinical } \\
\text { data, was inter/intra-rater } \\
\text { reliability reported? }\end{array}$ & $\begin{array}{l}\text { Prospective } \\
\text { study? }\end{array}$ & $\begin{array}{l}\text { Does PMR spectrum } \\
\text { appear realistic } \\
\text { according to } \\
\text { information } \\
\text { given? Did any } \\
\text { also have GCAs? }\end{array}$ & $\begin{array}{l}\text { Consecutive } \\
\text { selection of } \\
\text { participants? }\end{array}$ & $\begin{array}{l}\text { Comparator } \\
\text { condition(s): } \\
\text { realistic? }\end{array}$ & $\begin{array}{l}\text { Reference } \\
\text { standard; who } \\
\text { performed it, } \\
\text { when? }\end{array}$ & $\begin{array}{l}\text { Did all } \\
\text { participants } \\
\text { receive all } \\
\text { tests? }\end{array}$ & $\begin{array}{l}\text { Free from } \\
\text { incorporation } \\
\text { bias? }\end{array}$ & $\begin{array}{l}\text { Free from } \\
\text { diagnostic } \\
\text { review bias? }\end{array}$ & $\begin{array}{l}\text { participants } \\
\text { have index } \\
\text { test } \\
\text { before } \\
\text { receiving } \\
\text { glucocorticoid } \\
\text { treatment? }\end{array}$ \\
\hline \multicolumn{12}{|c|}{ Musculoskeletal ultrasound (MSK USS) } \\
\hline $\begin{array}{l}\text { Dasgupta } \\
\text { et } a l^{15 *} \dagger\end{array}$ & $\begin{array}{l}\text { MSK USS } \\
\text { shoulders, } \\
\text { hips }\end{array}$ & $\begin{array}{l}\text { Rheumatologist or radiologist, } \\
\text { one per site; reliability } \\
\text { reported separately (Scheel } \\
\text { et al, 2009); at some sites } \\
\text { sonographer was clinical } \\
\text { assessor }\end{array}$ & Yes & Yes; none had GCA & No & $\begin{array}{l}\text { Yes: }>50 \text { years, } \\
<12 \text { weeks' } \\
\text { history of } \\
\text { bilateral } \\
\text { shoulder pain, } \\
\text { not felt to be } \\
\text { PMR }\end{array}$ & $\begin{array}{l}\text { Clinical } \\
\text { diagnosis; by } \\
\text { investigator; } \\
\text { after } 6 \text { months }\end{array}$ & $\begin{array}{l}5 \text { PMR and } \\
15 \text { controls } \\
\text { did not have } \\
\text { scans }\end{array}$ & $\begin{array}{l}\text { Yes- } \\
\text { diagnosis } \\
\text { made before } \\
\text { USS, and } \\
\text { assessors told } \\
\text { not to use } \\
\text { USS findings } \\
\text { in making } \\
\text { diagnosis }\end{array}$ & $\begin{array}{l}\text { Sonographer } \\
\text { and clinical } \\
\text { assessor } \\
\text { were } \\
\text { sometimes } \\
\text { same person }\end{array}$ & Yes \\
\hline $\begin{array}{l}\text { Ruta } \\
\text { et } a^{\beta{ }^{*}} \dagger\end{array}$ & $\begin{array}{l}\text { MSK USS } \\
\text { shoulders }\end{array}$ & $\begin{array}{l}\text { Single } \\
\text { rheumatologist-sonographer } \\
\text { blinded to clinical data; } \\
\text { reliability not reported }\end{array}$ & Yes & $\begin{array}{l}\text { Maybe: relapsing PMR } \\
\text { (new-onset bilateral painful } \\
\text { shoulder and prior } \\
\text { diagnosis PMR); none had } \\
\text { GCA }\end{array}$ & Yes & $\begin{array}{l}\text { Maybe: } \\
\text { relapsing RA } \\
\text { (new-onset } \\
\text { bilateral painful } \\
\text { shoulder and } \\
\text { prior diagnosis } \\
\text { of RA) }\end{array}$ & $\begin{array}{l}\text { PMR: clinical } \\
\text { diagnosis } \\
\text { +Healey criteria; } \\
\text { RA: ACR } 2010 \\
\text { criteria; by } \\
\text { treating } \\
\text { rheumatologist }\end{array}$ & Yes & Yes & Yes & $\begin{array}{l}\mathrm{No} \text {; were on } \\
\leq 10 \mathrm{mg} \\
\text { prednisolone; } \\
\text { most were on } \\
2-4 \mathrm{mg} ; \\
\text { treatment did } \\
\text { not seem to } \\
\text { affect USS } \\
\text { findings }\end{array}$ \\
\hline $\begin{array}{l}\text { Falsetti } \\
\text { et } a l^{44} \dagger\end{array}$ & $\begin{array}{l}\text { MSK USS at } \\
\text { multiple } \\
\text { sites }\end{array}$ & $\begin{array}{l}\text { Single } \\
\text { rheumatologist-sonographer, } \\
\text { not blinded to clinical data; } \\
\text { reliability not reported }\end{array}$ & Yes & \multicolumn{3}{|c|}{$\begin{array}{l}\text { Yes: all participants referred from primary care with } \\
\text { polymyalgic syndrome fulfilling Bird criteria; one developed } \\
\text { GCA later. All participants drawn from this same population } \\
\text { (single-gate study design). } 29 / 61 \text { ( } 47.5 \% \text { ) had final diagnosis } \\
\text { PMR. Many of those with RA were seropositive }\end{array}$} & $\begin{array}{l}\text { Clinical } \\
\text { diagnosis; by } 2 \\
\text { rheumatologists, } \\
\text { after } 1 \text { year }\end{array}$ & Yes & No & No & Yes \\
\hline $\begin{array}{l}\text { Cantini } \\
\text { et } a{ }^{\beta 4}\end{array}$ & $\begin{array}{l}\text { MSK USS } \\
\text { hips and } \\
\text { MRI pelvic } \\
\text { girdle }\end{array}$ & $\begin{array}{l}\text { Two radiologists for each test } \\
\text { (unclear whether these were } \\
\text { same people), unclear } \\
\text { whether blinded to clinical } \\
\text { data (note alternating } \\
\text { recruitment of cases/2 } \\
\text { controls); reliability not } \\
\text { reported }\end{array}$ & Yes & $\begin{array}{l}\text { A subset: PMR with pelvic } \\
\text { girdle involvement; } 3 \text { also } \\
\text { had biopsy-proven GCA; } \\
\text { none developed RA (1987 } \\
\text { ACR criteria) after average } \\
\text { follow-up } 26 \text { months }\end{array}$ & Yes & $\begin{array}{l}\text { Maybe: next } 2 \\
\text { consecutive } \\
\text { outpatients } \\
>50 \text { years with } \\
\text { active rheumatic } \\
\text { disease (RA/ } \\
\text { PsA/OA) and } \\
\text { bilateral hip } \\
\text { ache }\end{array}$ & $\begin{array}{l}\text { Clinical } \\
\text { diagnosis } \\
\text { +Healey criteria } \\
\text { PMR, followed } \\
\text { up to ensure no } \\
\text { evolution to RA }\end{array}$ & $\begin{array}{l}\text { Only } 10 \text { of } \\
40 \text { controls } \\
\text { had MRI } \\
\text { (unclear how } \\
\text { these were } \\
\text { selected) }\end{array}$ & Yes & Unclear & $\begin{array}{l}\text { Yes for PMR; } \\
\text { unclear for } \\
\text { controls }\end{array}$ \\
\hline $\begin{array}{l}\text { Frediani } \\
2002 \dagger^{45}\end{array}$ & $\begin{array}{l}\text { MSK USS at } \\
\text { multiple } \\
\text { sites }\end{array}$ & $\begin{array}{l}\text { Two rheumatologist- } \\
\text { sonographers, blinded to } \\
\text { diagnosis; "medium rates } \\
\text { concordance [agreement]" } \\
\text { reported but no test statistics } \\
\text { quoted }\end{array}$ & Yes & $\begin{array}{l}\text { Yes: "PMR patients with a } \\
\text { relatively certain } \\
\text { diagnosis"-Healey } \\
\text { criteria; } 2 \text { also had GCA }\end{array}$ & Yes & $\begin{array}{l}\text { No: RA (ARA } \\
1987 \text { criteria); } \\
\text { SpA (ESSG } \\
\text { criteria) }\end{array}$ & $\begin{array}{l}\text { Clinical } \\
\text { diagnosis } \\
\text { +Healey criteria } \\
\text { PMR; 2-year } \\
\text { follow-up to } \\
\text { confirm } \\
\text { diagnosis }\end{array}$ & Yes & Yes & $\begin{array}{l}\text { No, but } \\
\text { diagnosis not } \\
\text { changed } \\
\text { after USS }\end{array}$ & Yes \\
\hline
\end{tabular}




\begin{tabular}{|c|c|c|c|c|c|c|c|c|c|c|c|}
\hline Study & $\begin{array}{l}\text { Index test: } \\
\text { imaging } \\
\text { modality }\end{array}$ & $\begin{array}{l}\text { Who performed index test, } \\
\text { were they blinded to clinical } \\
\text { data, was inter/intra-rater } \\
\text { reliability reported? }\end{array}$ & $\begin{array}{l}\text { Prospective } \\
\text { study? }\end{array}$ & $\begin{array}{l}\text { Does PMR spectrum } \\
\text { appear realistic } \\
\text { according to } \\
\text { information } \\
\text { given? Did any } \\
\text { also have GCAs? }\end{array}$ & $\begin{array}{l}\text { Consecutive } \\
\text { selection of } \\
\text { participants? }\end{array}$ & $\begin{array}{l}\text { Comparator } \\
\text { condition(s): } \\
\text { realistic? }\end{array}$ & $\begin{array}{l}\text { Reference } \\
\text { standard; who } \\
\text { performed it, } \\
\text { when? }\end{array}$ & $\begin{array}{l}\text { Did all } \\
\text { participants } \\
\text { receive all } \\
\text { tests? }\end{array}$ & $\begin{array}{l}\text { Free from } \\
\text { incorporation } \\
\text { bias? }\end{array}$ & $\begin{array}{l}\text { Free from } \\
\text { diagnostic } \\
\text { review bias? }\end{array}$ & $\begin{array}{l}\text { Did } \\
\text { participants } \\
\text { have index } \\
\text { test } \\
\text { before } \\
\text { receiving } \\
\text { glucocorticoid } \\
\text { treatment? }\end{array}$ \\
\hline $\begin{array}{l}\text { Cantini } \\
\text { et } a l^{\beta 3}\end{array}$ & $\begin{array}{l}\text { MSK USS } \\
\text { shoulders }\end{array}$ & $\begin{array}{l}\text { Two radiologists together, } \\
\text { blinded to clinical diagnosis } \\
\text { (but note recruitment of } 2 \\
\text { controls after each case); } \\
\text { reliability not reported }\end{array}$ & Yes & $\begin{array}{l}\text { Yes: }>1 \text { month pain neck } \\
\text { and shoulder girdle; } \\
\text { morning stiffness }>1 \mathrm{~h} \text {; } \\
\text { ESR }>40 ; 5 \text { also had } \\
\text { biopsy proven GCA; } \\
\text { follow-up for mean } \\
8 \text { months to exclude those } \\
\text { fulfilling } 1987 \text { ARA RA } \\
\text { criteria }\end{array}$ & Yes & $\begin{array}{l}\text { Maybe: next } 2 \\
\text { consecutive } \\
\text { outpatients } \\
>50 \text { years with } \\
\text { bilateral } \\
\text { shoulder aching, } \\
\text { stiffness (RA/ } \\
\text { PsA/SpA/OA/ } \\
\text { FM/CTD) }\end{array}$ & $\begin{array}{l}\text { Clinical } \\
\text { diagnosis } \\
\text { + Healey criteria; } \\
\text { by } 1 \text { of } 4 \\
\text { rheumatologists; } \\
\text { follow-up to } \\
\text { confirm } \\
\text { diagnosis }\end{array}$ & Yes & Yes & $\begin{array}{l}\text { Unclear; but } \\
\text { participant } \\
\text { selection } \\
\text { protocol } \\
\text { implies } \\
\text { participants } \\
\text { did not } \\
\text { switch } \\
\text { between } \\
\text { case/control } \\
\text { groups }\end{array}$ & $\begin{array}{l}\text { Yes for PMR, } \\
\text { unclear for } \\
\text { controls }\end{array}$ \\
\hline Coari et $a{ }^{\beta 8}$ & $\begin{array}{l}\text { MSK USS } \\
\text { shoulders }\end{array}$ & $\begin{array}{l}\text { Two } \\
\text { rheumatologist-sonographers, } \\
\text { unclear whether blinded to } \\
\text { clinical data; reliability not } \\
\text { reported }\end{array}$ & $\begin{array}{l}\text { Not stated } \\
\text { but implied }\end{array}$ & $\begin{array}{l}\text { No: treated PMR; not } \\
\text { stated whether any had } \\
\text { GCA }\end{array}$ & Not stated & $\begin{array}{l}\text { No: treated; } \\
\text { one-third of RA } \\
\text { patients erosive }\end{array}$ & $\begin{array}{l}\text { Clinical } \\
\text { diagnosis (ARA } \\
1987 \text { for RA); } \\
\text { not stated by } \\
\text { whom or } \\
\text { whether followed } \\
\text { up }\end{array}$ & $\begin{array}{l}\text { Only PMR } \\
\text { each had } \\
\text { both } \\
\text { shoulders } \\
\text { scanned; } \\
\text { unit of } \\
\text { analysis was } \\
\text { shoulder not } \\
\text { patient }\end{array}$ & Yes & Unclear & No \\
\hline $\begin{array}{l}\text { Lange } \\
\text { et } a A^{46}\end{array}$ & $\begin{array}{l}\text { MSK USS } \\
\text { shoulders }\end{array}$ & $\begin{array}{l}\text { Not stated; reliability not } \\
\text { reported }\end{array}$ & $\begin{array}{l}\text { Not stated } \\
\text { but implied }\end{array}$ & $\begin{array}{l}\text { Yes: }>60 \text { years, pain and } \\
\text { several hours' morning } \\
\text { stiffness of shoulders, } \\
\text { neck and/or pelvic girdle, } \\
\text { limited motion in neck and } \\
\text { shoulder, ESR }>45 \text {, } \\
\text { response to prednisolone } \\
30 \text { mg or less); } 6 \text { had } \\
\text { headache, } 2 \text { had } \\
\text { biopsy-proven GCA }\end{array}$ & Not stated & $\begin{array}{l}\text { Maybe: "initially } \\
\text { had similar } \\
\text { complaints (to } \\
\text { the PMR cases) } \\
\ldots \text { involvement } \\
\text { of arthritis in } \\
\text { additional joints } \\
\text { and bony } \\
\text { erosions" }\end{array}$ & $\begin{array}{l}\text { Clinical } \\
\text { diagnosis; not } \\
\text { stated by whom } \\
\text { or whether } \\
\text { followed up }\end{array}$ & Yes & $\begin{array}{l}\text { Yes (implied } \\
\text { but not stated) }\end{array}$ & Unclear & $\begin{array}{l}\text { Yes (implied } \\
\text { but not stated) }\end{array}$ \\
\hline $\begin{array}{l}\text { Lange } \\
\text { et } a A^{77}\end{array}$ & $\begin{array}{l}\text { MSK USS } \\
\text { shoulders }\end{array}$ & $\begin{array}{l}\text { Not stated; reliability not } \\
\text { reported }\end{array}$ & $\begin{array}{l}\text { Not stated } \\
\text { but implied }\end{array}$ & $\begin{array}{l}\text { Yes: }>60 \text { years, pain and } \\
\text { several hours' morning } \\
\text { stiffness of shoulders, } \\
\text { neck and/or pelvic girdle, } \\
>4 \text { weeks duration } \\
\text { symptoms, ESR }>45 \text {, } \\
\text { response to prednisolone } \\
30 \text { mg or less); } 5 \text { had } \\
\text { headache, } 4 \text { had } \\
\text { biopsy-proven GCA }\end{array}$ & Not stated & $\begin{array}{l}\text { Maybe: "initially } \\
\text { had similar } \\
\text { complaints (to } \\
\text { the PMR cases) } \\
\text {... involvement } \\
\text { of arthritis in } \\
\text { additional joints } \\
\text { and bony } \\
\text { erosions" }\end{array}$ & $\begin{array}{l}\text { Clinical } \\
\text { diagnosis; not } \\
\text { stated by whom } \\
\text { or whether } \\
\text { followed up }\end{array}$ & Yes & $\begin{array}{l}\text { Yes (implied } \\
\text { but not stated) }\end{array}$ & Unclear & $\begin{array}{l}\text { Yes (implied } \\
\text { but not stated) }\end{array}$ \\
\hline $\begin{array}{l}\text { Macchioni } \\
\text { et } a P^{3}\end{array}$ & $\begin{array}{l}\text { MSK USS } \\
\text { shoulders, } \\
\text { hips }\end{array}$ & $\begin{array}{l}\text { Single } \\
\text { rheumatologist-sonographer; } \\
\text { blinding to clinical data not } \\
\text { stated; reliability not reported }\end{array}$ & No & $\begin{array}{l}\text { Yes: patients seen with } \\
\text { suspected PMR; patients } \\
\text { with GCA excluded }\end{array}$ & Yes & $\begin{array}{l}\text { No: patients in } \\
\text { early arthritis } \\
\text { clinic; no } \\
\text { requirement for } \\
\text { comparable } \\
\text { symptoms }\end{array}$ & $\begin{array}{l}\text { Clinical } \\
\text { diagnosis; } \\
\text { confirmed at } \\
1 \text { year by } 2 \text { lead } \\
\text { authors }\end{array}$ & Yes & Unclear & No & Yes \\
\hline
\end{tabular}

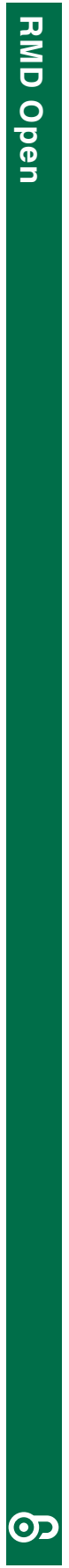

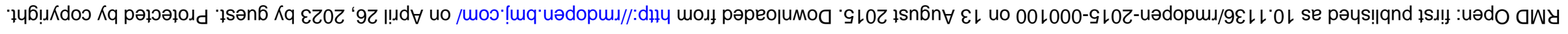




\begin{tabular}{|c|c|c|c|c|c|c|c|c|c|c|c|}
\hline Study & $\begin{array}{l}\text { Index test: } \\
\text { imaging } \\
\text { modality }\end{array}$ & $\begin{array}{l}\text { Who performed index test, } \\
\text { were they blinded to clinical } \\
\text { data, was inter/intra-rater } \\
\text { reliability reported? }\end{array}$ & $\begin{array}{l}\text { Prospective } \\
\text { study? }\end{array}$ & $\begin{array}{l}\text { Does PMR spectrum } \\
\text { appear realistic } \\
\text { according to } \\
\text { information } \\
\text { given? Did any } \\
\text { also have GCAs? }\end{array}$ & $\begin{array}{l}\text { Consecutive } \\
\text { selection of } \\
\text { participants? }\end{array}$ & $\begin{array}{l}\text { Comparator } \\
\text { condition(s): } \\
\text { realistic? }\end{array}$ & $\begin{array}{l}\text { Reference } \\
\text { standard; who } \\
\text { performed it, } \\
\text { when? }\end{array}$ & $\begin{array}{l}\text { Did all } \\
\text { participants } \\
\text { receive all } \\
\text { tests? }\end{array}$ & $\begin{array}{l}\text { Free from } \\
\text { incorporation } \\
\text { bias? }\end{array}$ & $\begin{array}{l}\text { Free from } \\
\text { diagnostic } \\
\text { review bias? }\end{array}$ & $\begin{array}{l}\text { Did } \\
\text { participants } \\
\text { have index } \\
\text { test } \\
\text { before } \\
\text { receiving } \\
\text { glucocorticoid } \\
\text { treatment? }\end{array}$ \\
\hline \multicolumn{12}{|l|}{ MRI } \\
\hline $\begin{array}{l}\text { Salvarani } \\
\text { et } a \beta^{7}\end{array}$ & $\begin{array}{l}1.5 \mathrm{~T} \text { MRI } \\
\text { lumbar spine } \\
\text { (bursitis) }\end{array}$ & $\begin{array}{l}\text { Radiologist; blinded to clinical } \\
\text { findings and diagnosis; } \\
\text { reliability not reported }\end{array}$ & Yes & $\begin{array}{l}\text { A subset: PMR by Chuang } \\
\text { criteria+pelvic girdle } \\
\text { symptoms; none had GCA }\end{array}$ & Yes & $\begin{array}{l}\text { Maybe: treated } \\
\text { patients with } \\
\text { lumbar pain } \\
(\mathrm{SpA} / \mathrm{OA} / \mathrm{RA})\end{array}$ & $\begin{array}{l}\text { Clinical } \\
\text { diagnosis } \\
\text { +Chuang } \\
\text { criteria, followed } \\
\text { up for 10-16 } \\
\text { months to } \\
\text { exclude RA } \\
\text { (ARA 1987) or } \\
\text { other conditions }\end{array}$ & Yes & Yes & Yes & $\begin{array}{l}\text { Yes for PMR, } \\
\text { unclear for } \\
\text { controls }\end{array}$ \\
\hline $\begin{array}{l}\text { Cimmino } \\
\text { et } a f^{40}\end{array}$ & $\begin{array}{l}0.2 \mathrm{~T} \text { MRI } \\
\text { hands } \\
\text { (extremity } \\
\text { MRI)- } \\
\text { tenosynovitis }\end{array}$ & $\begin{array}{l}\text { Two rheumatologists and one } \\
\text { PhD, blinded to diagnosis; } \\
\text { reliability not reported but } \\
\text { Parodi et al } 2006 \text { quoted in } \\
\text { support }\end{array}$ & Yes & $\begin{array}{l}\text { Yes: PMR by Chuang } \\
\text { criteria; none had GCA }\end{array}$ & $\begin{array}{l}\text { Yes for PMR, } \\
\text { not for } \\
\text { controls }\end{array}$ & $\begin{array}{l}\text { No: Healthy } \\
\text { controls of } \\
\text { similar ages, no } \\
\text { mention of } \\
\text { symptoms }\end{array}$ & $\begin{array}{l}\text { Clinical } \\
\text { diagnosis } \\
\text { +Chuang } \\
\text { criteria, followed } \\
\text { for } 8-124 \\
\text { months to } \\
\text { exclude GCA, } \\
\text { RA and other } \\
\text { erosive disease }\end{array}$ & $\begin{array}{l}\text { Yes but } 4 \\
\text { hands could } \\
\text { not be } \\
\text { interpreted }\end{array}$ & Yes & Yes & Yes \\
\hline $\begin{array}{l}\text { Salvarani } \\
\text { et } a^{\beta 6}\end{array}$ & $\begin{array}{l}1 \mathrm{~T} \mathrm{MRI} \\
\text { cervical } \\
\text { spine } \\
\text { (bursitis) }\end{array}$ & $\begin{array}{l}\text { One radiologist, blinded to } \\
\text { clinical data and diagnosis } \\
\text { (but note alternating } \\
\text { recruitment of cases, } \\
\text { controls); reliability not } \\
\text { reported }\end{array}$ & Yes & $\begin{array}{l}\text { Yes: PMR (reference } \\
\text { Salvarani review 2002); } \\
\text { none had GCA }\end{array}$ & Yes & $\begin{array}{l}\text { No: Next } \\
\text { patients with } \\
\text { neck pain seen } \\
\text { after PMR } \\
\text { patients }\end{array}$ & $\begin{array}{l}\text { Clinical } \\
\text { diagnosis } \\
\text { +criteria; } \\
\text { followed for } \\
10-16 \text { months to } \\
\text { exclude other } \\
\text { conditions }\end{array}$ & Yes & Yes & Yes & Yes \\
\hline $\begin{array}{l}\text { Marzo } \\
\text { et } \mathrm{al}^{\prime{ }^{3}}\end{array}$ & $\begin{array}{l}1.5 \mathrm{~T} \text { MRI of } \\
\text { most } \\
\text { swollen } \\
\text { hand }\end{array}$ & $\begin{array}{l}\text { One assessor per MRI } \\
\text { feature, blinded to clinical } \\
\text { data; reliability not reported }\end{array}$ & Yes & $\begin{array}{l}\text { No: Bird criteria+MCP joint } \\
\text { swelling }\end{array}$ & $\begin{array}{l}\text { Yes for RA, } \\
\text { not stated for } \\
\text { PMR }\end{array}$ & $\begin{array}{l}\text { No: ARA } 1987 \\
\text { criteria+MCP } \\
\text { joint swelling }\end{array}$ & $\begin{array}{l}\text { Clinical } \\
\text { diagnosis+Bird } \\
\text { criteria; followed } \\
\text { for mean of } \\
6 \text { years }\end{array}$ & Yes & Yes & Yes & $\begin{array}{l}\text { Yes except for } \\
\text { one PMR } \\
\text { patient }\end{array}$ \\
\hline $\begin{array}{l}\text { McGonagle } \\
\text { et } a 1^{12}\end{array}$ & $\begin{array}{l}1.5 \mathrm{~T} \mathrm{MRI} \\
\text { shoulder }\end{array}$ & $\begin{array}{l}\text { Two radiologists, blinded to } \\
\text { clinical data; reliability not } \\
\text { reported }\end{array}$ & Yes & $\begin{array}{l}\text { Yes: untreated PMR and } \\
\text { bilateral shoulder disease } \\
\text { without peripheral } \\
\text { arthropathy }\end{array}$ & No & $\begin{array}{l}\text { No: early RA } \\
\text { fulfilling } 1987 \\
\text { ARA criteria }\end{array}$ & $\begin{array}{l}\text { Clinical } \\
\text { diagnosis; no } \\
\text { follow-up } \\
\text { reported to } \\
\text { exclude other } \\
\text { conditions }\end{array}$ & $\begin{array}{l}\text { Only } 6 / 14 \\
\text { PMR } \\
\text { patients had } \\
\text { both } \\
\text { shoulders } \\
\text { imaged }\end{array}$ & Yes & Yes & $\begin{array}{l}\text { Yes for PMR; } \\
\text { not for 8/14 RA }\end{array}$ \\
\hline $\begin{array}{l}\text { Salvarani } \\
\text { et } a f^{48}\end{array}$ & $\begin{array}{l}0.5 \mathrm{~T} \text { MRI } \\
\text { shoulder }\end{array}$ & $\begin{array}{l}\text { One radiologist, blinded to } \\
\text { clinical data and diagnosis; } \\
\text { reliability not reported }\end{array}$ & Yes & $\begin{array}{l}\text { Yes: Healey criteria PMR; } \\
\text { none had GCA }\end{array}$ & Unclear & $\begin{array}{l}\text { No: } \\
\text { elderly-onset RA } \\
\text { by modified } \\
1987 \text { ARA } \\
\text { criteria, with } \\
\text { clinical evidence } \\
\text { shoulder } \\
\text { involvement }\end{array}$ & $\begin{array}{l}\text { Clinical } \\
\text { diagnosis } \\
\text { +Healey criteria; } \\
\text { no follow-up } \\
\text { reported to } \\
\text { exclude other } \\
\text { conditions }\end{array}$ & $\begin{array}{l}\text { The first } 4 \\
\text { PMR had } \\
\text { both } \\
\text { shoulders } \\
\text { imaged; after } \\
\text { that only one } \\
\text { shoulder }\end{array}$ & Yes & Yes & Yes \\
\hline
\end{tabular}

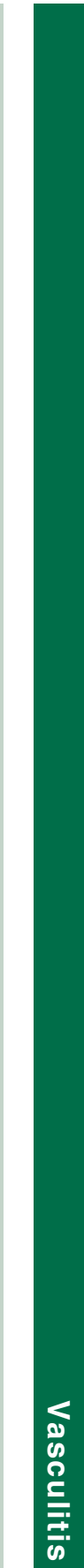

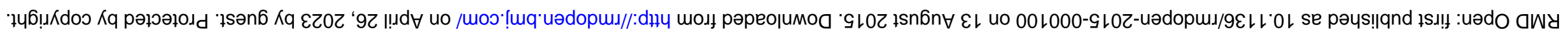




\begin{tabular}{|c|c|c|c|c|c|c|c|c|c|c|c|}
\hline Study & $\begin{array}{l}\text { Index test: } \\
\text { imaging } \\
\text { modality }\end{array}$ & $\begin{array}{l}\text { Who performed index test, } \\
\text { were they blinded to clinical } \\
\text { data, was inter/intra-rater } \\
\text { reliability reported? }\end{array}$ & $\begin{array}{l}\text { Prospective } \\
\text { study? }\end{array}$ & $\begin{array}{l}\text { Does PMR spectrum } \\
\text { appear realistic } \\
\text { according to } \\
\text { information } \\
\text { given? Did any } \\
\text { also have GCAs? }\end{array}$ & $\begin{array}{l}\text { Consecutive } \\
\text { selection of } \\
\text { participants? }\end{array}$ & $\begin{array}{l}\text { Comparator } \\
\text { condition(s): } \\
\text { realistic? }\end{array}$ & $\begin{array}{l}\text { Reference } \\
\text { standard; who } \\
\text { performed it, } \\
\text { when? }\end{array}$ & $\begin{array}{l}\text { Did all } \\
\text { participants } \\
\text { receive all } \\
\text { tests? }\end{array}$ & $\begin{array}{l}\text { Free from } \\
\text { incorporation } \\
\text { bias? }\end{array}$ & $\begin{array}{l}\text { Free from } \\
\text { diagnostic } \\
\text { review bias? }\end{array}$ & $\begin{array}{l}\text { Did } \\
\text { participants } \\
\text { have index } \\
\text { test } \\
\text { before } \\
\text { receiving } \\
\text { glucocorticoid } \\
\text { treatment? }\end{array}$ \\
\hline \multicolumn{12}{|c|}{${ }^{18} \mathrm{~F}$-fluorodeoxyglucose-positron emission tomography (FDG-PET) } \\
\hline $\begin{array}{l}\text { Yamashita } \\
\text { et } a^{\beta 5}\end{array}$ & $\begin{array}{l}\text { FDG-PET/ } \\
\text { CT whole } \\
\text { body }\end{array}$ & $\begin{array}{l}\text { Not stated who reported test; } \\
\text { unclear whether blinded to } \\
\text { clinical info; reliability not } \\
\text { reported }\end{array}$ & No & $\begin{array}{l}\text { No: inpatients, having } \\
\text { PET/CT to exclude other } \\
\text { diseases for example, } \\
\text { suspected malignancy; } \\
\text { none had clinical evidence } \\
\text { GCA }\end{array}$ & Yes & $\begin{array}{l}\text { No (other } \\
\text { rheumatic } \\
\text { diseases with } \\
\text { suspected } \\
\text { malignancy; } \\
11 / 17 \mathrm{RA} \text { ) }\end{array}$ & $\begin{array}{l}\text { Clinical } \\
\text { diagnosis } \\
\text { +Chuang } \\
\text { +Healey criteria; } \\
\text { length of } \\
\text { follow-up not } \\
\text { specified }\end{array}$ & Yes & Unclear & No & $\begin{array}{l}\text { Yes for PMR, } \\
\text { not stated for } \\
\text { controls }\end{array}$ \\
\hline $\begin{array}{l}\text { Camellino } \\
\text { et } a f^{5} t\end{array}$ & $\begin{array}{l}\text { FDG-PET/ } \\
\text { CT }\end{array}$ & $\begin{array}{l}\text { Rheumatologist and } \\
\text { radiologist, blinded to clinical } \\
\text { data (pers comm); reliability } \\
\text { not reported }\end{array}$ & Yes & $\begin{array}{l}\text { Little information on how } \\
\text { patients were identified }\end{array}$ & Yes & $\begin{array}{l}\text { No }(65 \text { matched } \\
\text { controls with no } \\
\text { inflammatory } \\
\text { disease; } 10 \text { with } \\
\text { treated RA) }\end{array}$ & $\begin{array}{l}\text { Fulfilled Bird and } \\
\text { ACR/EULAR } \\
\text { criteria; median } \\
\text { follow-up } \\
22 \text { months }\end{array}$ & Yes & Yes & Probably & $\begin{array}{l}\text { Yes for PMR/ } \\
\text { controls, no for } \\
\text { RA }\end{array}$ \\
\hline $\begin{array}{l}\text { Takahasi } \\
\text { et } a P^{4} \dagger\end{array}$ & $\begin{array}{l}\text { FDG-PET/ } \\
\text { CT }\end{array}$ & $\begin{array}{l}\text { Radiologists, blinded to } \\
\text { clinical data [pers comm]; } \\
\text { reliability not reported }\end{array}$ & No & $\begin{array}{l}\text { No: inpatients and } \\
\text { outpatients, having PET/ } \\
\text { CT to exclude other } \\
\text { diseases, for example, } \\
\text { suspected malignancy; } \\
\text { none had clinical evidence } \\
\text { of GCA }\end{array}$ & Yes & $\begin{array}{l}\text { Maybe } \\
\text { (untreated, } \\
\text { elderly-onset } \\
\text { RA) }\end{array}$ & $\begin{array}{l}\text { Diagnosed by } \\
\text { attending } \\
\text { doctors prior to } \\
\text { PET/CT (pers } \\
\text { comm); } \\
\text { diagnosis did not } \\
\text { change on } \\
\text { follow-up (pers } \\
\text { comm). and } \\
\text { verified by } \\
\text { classification } \\
\text { criteria }\end{array}$ & Yes & Yes & Yes & Yes \\
\hline \multicolumn{12}{|c|}{$\begin{array}{l}\text { The PET or PET/CT studies that did not report data extractable into } 2 \times 2 \text { table format are not listed here. Before-after or prognostic studies, if they did not report data extractable into } 2 \times 2 \text { table format, are not reported here. } \\
\text { Incorporation bias means where the imaging (index test) informs the diagnosis (reference standard). }\end{array}$} \\
\hline \multicolumn{12}{|c|}{ Diagnostic review bias means where the diagnosis (reference standard) was carried out or verified with knowledge of the imaging (index test). } \\
\hline
\end{tabular}


Table 3 Summary data for individual tests

\section{Anatomical finding}

Cervical interspinous bursitis

Cervical interspinous bursitis, comparator no inflammation

Lumbar interspinous bursitis

Lumbar interspinous bursitis, comparator no inflammation

Any interspinous bursitis

Subacromial bursitis on at least one side

Subacromial bursitis on at least one side: comparator RA

Subacromial bursitis on at least one side: comparator painful shoulder conditions Subacromial bursitis on both sides

Subacromial bursitis on both sides: comparator RA

Iliopsoas bursitis

Iliopectineal (iliopsoas) bursitis, comparator RA Ischiogluteal bursitis

Trochanteric bursitis on at least one side
Studies (imaging modality)

Salvarani et $a^{\beta 6}(\mathrm{MRI})$

Camellino et $a^{25}$ (PET/CT)

Salvarani et $a \beta^{37}$ (MRI)

Camellino et $a^{25}$ (PET/CT)

Yamashita et a ${ }^{\beta 5}$ (PET/CT)

Cantini et $a^{\beta 3}$ (USS)

Frediani et $a f^{45}$ (USS)

Falsetti et a/ ${ }^{44}$ (USS)

Dasgupta et al ${ }^{15}$ (USS)

Cantini et $a{ }^{\beta 3}$ (USS)*

Frediani et $a{ }^{45}$ (USS)

Falsetti et $a f^{44}$ (USS)

Dasgupta et al ${ }^{15}$ (USS)

Salvarani et $a f^{48}$ (MRI)

Coari et $a^{\beta 8}$ (USS)

Dasgupta et $\mathrm{al}^{15}$ (USS)

Ruta et $a^{\beta 9}$ (USS)

Dasgupta et al ${ }^{15}$ (USS)

Ruta et $a^{\beta 9}$ (USS)

Cantini et $a \beta^{33}$ (USS)

Frediani et $a f^{45}$ (USS)

Falsetti et $a f^{44}$ (USS)

Dasgupta et al ${ }^{15}$ (USS)

Cantini et $a{ }^{\beta 3}$ (USS)*

Frediani et $\mathrm{al}^{45}$ (USS)

Falsetti et $a^{44}$ (USS)

Dasgupta et al ${ }^{15}$ (USS)

Dasgupta et al ${ }^{15}$ (USS)

Ruta et $a{ }^{\beta 9}$ (USS)

Cantini et $a l^{\beta 4}$ (MRI)

Cantini et $a^{\beta 4}$ (USS)

Takahashi et a ${ }^{4}$

Cantini et $a^{\beta 4}$ (MRI)

Yamashita et a ${ }^{35}$ (PET/CT)

Cantini et $a \beta^{34}$ (USS)

Cantini et $a^{\beta 4}$ (USS)

Cantini et $a^{\beta 4}$ (MRI)

Dasgupta et al ${ }^{15}$ (USS)

Yamashita et a ${ }^{35}$ (PET/CT)

\section{Sensitivity}

(95\% Cl), \%

$0.83(0.55$ to 0.95$)$

$0.10(0.05$ to 0.19$)$

0.60 (0.31 to 0.83$)$

0.46 (0.35 to 0.48$)$

0.79 (0.52 to 0.92$)$ 0.96 (0.88 to 0.99$)$ 0.70 (0.56 to 0.81$)$

$0.79(0.62$ to 0.90$)$

0.56 (0.47 to 0.65$)$

0.80 (0.55 to 0.93$)$

$0.96(0.73$ to 1.00$)$ 0.09 (0.03 to 0.24$)$ $0.56(0.47$ to 0.65$)$ $0.73(0.56$ to 0.86$)$ $0.56(0.47$ to 0.65$)$ 0.55 (0.43 to 0.67$)$ 0.93 (0.83 to 0.97$)$ 0.54 (0.40 to 0.67 ) $0.69(0.51$ to 0.83$)$ $0.32(0.24$ to 0.41$)$ 0.66 (0.36 to 0.87$)$

0.75 (0.44 to 0.92$) \quad 3.86$ (1.3 to 11$)$ 0.90 (0.84 to 0.94$) \quad 0.95(0.29$ to 3.2$)$ $0.72(0.57$ to 0.83$) \quad 2.0(1.2$ to 3.2$)$ 0.67 (0.49 to 0.81$) \quad 2.2(1.3$ to 3.8$)$ 0.70 (0.55 to 0.81$) \quad 1.9(1.2$ to 2.9$)$ 0.75 (0.63 to 0.84$) \quad 2.2(1.3$ to 3.6$)$ 0.99 (0.95 to 1.00$) \quad 106(15$ to 747$)$ 0.68 (0.58 to 0.76$) \quad 1.7(1.1$ to 2.5$)$ 0.78 (0.61 to 0.89$) \quad 3.2(1.6$ to 6.3$)$ 0.88 (0.81 to 0.92$) \quad 2.6(1.6$ to 4.2$)$ 0.89 (0.66 to 0.97$) \quad 6.2(1.2$ to 32$)$

$0.32(0.24$ to 0.41$)$ $0.37(0.22$ to 0.55$)$ $0.50(0.30$ to 0.70$)$ $0.30(0.15$ to 0.50$)$ 0.59 (0.41 to 0.75$)$ 0.25 (0.11 to 0.47$)$ 0.86 (0.60 to 0.96$)$ $0.20(0.081$ to 0.42$)$ $0.98(0.81$ to 1.00$)$ $0.98(0.81$ to 1.00$)$ $0.21(0.15$ to 0.30$)$ 0.71 (0.45 to 0.88$)$

\section{Specificity} ratio $(95 \% \mathrm{Cl})$

$0.99(0.93$ to 1.00$) \quad 13(0.8$ to 226$)$

$0.91(0.62$ to 0.98$) \quad 6.6(1.0$ to 46$)$

$0.82(0.59$ to 0.94$) \quad 4.5(1.5$ to 13$)$ $0.78(0.70$ to 0.85$) \quad 4.4(3.1$ to 6.2$)$ $0.61(0.51$ to 0.70$) \quad 1.8(1.3$ to 2.4$)$ 0.59 (0.42 to 0.74$) \quad 2.0(1.2$ to 3.1$)$ 0.65 (0.58 to 0.72$) \quad 1.6(1.2$ to 2.1$)$ $1.6(1.2$ to 2.1$)$
$2.5(1.6$ to 3.8$)$ 0.97 (0.83 to 0.99$)$ 0.80 (0.50 to 0.94$)$ $0.90(0.77$ to 0.96$)$ 0.90 (0.60 to 0.98$)$ 0.90 (0.60 to 0.98$)$ $0.95(0.84$ to 0.99$)$

0.70 (0.54 to 0.81$)$

0.78 (0.48 to 0.93$)$

$0.91(0.84$ to 0.95$)$ 0.88 (0.66 to 0.97$)$
Negative likelihood ratio $(95 \% \mathrm{Cl})$

0.24 (0.065 to 0.90$)$ 0.9 (0.91 to 0.99$)$

$0.4(0.20$ to 0.96$)$ 0.54 (0.43 to 0.68$)$

$0.26(0.093$ to 0.73$)$ 0.04 (0.01 to 0.18$)$ $0.49(0.31$ to 0.77$)$ $0.35(0.16$ to 0.75$)$ 0.67 (0.53 to 0.85$)$ 0.30 (0.11 to 0.81$)$

$0.05(0.003$ to 0.74$)$ 1.01 (0.89 to 1.1$)$ $0.61(0.46$ to 0.80$)$ $0.40(0.21$ to 0.76$)$ 0.63 (0.48 to 0.83$)$ 0.60 (0.44 to 0.82$)$ 0.07 (0.028 to 0.18$)$ 0.68 (0.49 to 0.94$)$ $0.40(0.22$ to 0.70$)$ 0.78 (0.68 to 0.89$)$ 0.38 (0.15 to 0.97$)$

0.78 (0.64 to 0.88$)$ 0.76 (0.53 to 0.90$)$
$1.5(0.8$ to 2.7$)$

11 (1.5 to 80$)$

2.5 (0.67 to 9.3$)$

3.0 (0.95 to 9.4$)$

$5.9(0.90$ to 39$)$

$2.5(0.34$ to 19$)$

3.6 (1.5 to 8.8$)$

$4.0(0.80$ to 20$)$

3.2 (2.0 to 5.1 )

4.3 (1.4 to 13$)$

2.3 (1.2 to 4.5$)$

6.1 (1.6 to 23 )
0.87 (0.64 to 0.88$)$ 0.66 (0.83 to 0.99$)$ 0.63 (0.37 to 1.1$)$

0.78 (0.57 to 1.1$)$

0.45 (0.28 to 0.75$)$

0.83 (0.60 to 1.2)

0.19 (0.05 to 0.69$)$

0.84 (0.70 to 1.1$)$

$0.03(0.002$ to 0.53$)$

0.87 (0.78 to 0.97 )
0.031 (0.002 to 0.49 )

0.32 (0.14 to 0.76$)$ 


\begin{tabular}{|c|c|c|c|c|c|}
\hline Anatomical finding & Studies (imaging modality) & $\begin{array}{l}\text { Sensitivity } \\
(95 \% \mathrm{Cl}), \%\end{array}$ & $\begin{array}{l}\text { Specificity } \\
(95 \% \mathrm{Cl}), \%\end{array}$ & $\begin{array}{l}\text { Positive likelihood } \\
\text { ratio }(95 \% \mathrm{Cl})\end{array}$ & $\begin{array}{l}\text { Negative likelihood } \\
\text { ratio }(95 \% \mathrm{Cl})\end{array}$ \\
\hline Hand extracapsular: comparator RA & Marzo-Ortega et al ${ }^{13}$ (MRI) & $0.80(0.49$ to 0.94$)$ & 0.80 (0.49 to 0.94$)$ & $4.0(1.1$ to 14$)$ & 0.25 (0.07 to 0.90$)$ \\
\hline Shoulder extracapsular: comparator RA & McGonagle et al ${ }^{12}$ (MRI) & $0.64(0.39$ to 0.84$)$ & $0.86(0.60$ to 0.96$)$ & 4.5 (1.2 to 17$)$ & $0.42(0.2$ to 0.87$)$ \\
\hline Long head biceps tenosynovitis on at least one & Cantini et $a^{\beta 3}$ (USS) & 0.81 (0.70 to 0.89$)$ & 0.47 (0.38 to 0.57$)$ & 1.5 (1.2 to 1.9$)$ & 0.41 (0.23 to 0.72$)$ \\
\hline \multirow[t]{2}{*}{ side } & Dasgupta et al ${ }^{15}$ (USS) & $0.66(0.57$ to 0.74$)$ & 0.54 (0.46 to 0.61$)$ & $1.4(1.2$ to 1.8$)$ & $0.63(0.47$ to 0.85$)$ \\
\hline & Frediani et $a{ }^{45}$ (USS) & 0.68 (0.54 to 0.79$)$ & 0.59 (0.49 to 0.68$)$ & 1.7 (1.2 to 2.2$)$ & 0.54 (0.35 to 0.84$)$ \\
\hline \multirow{9}{*}{$\begin{array}{l}\text { Long head biceps tenosynovitis on at least one } \\
\text { side: comparator RA }\end{array}$} & Coari et $a^{\beta 8}$ (USS) & $0.16(0.07$ to 0.32$)$ & $0.48(0.38$ to 0.58$)$ & 0.30 (0.13 to 0.69$)$ & 1.8 (1.4 to 2.3$)$ \\
\hline & Dasgupta et $a l^{15}$ (USS) & $0.66(0.57$ to 0.74$)$ & $0.44(0.31$ to 0.59$)$ & $1.2(0.89$ to 1.6$)$ & $0.76(0.51$ to 1.2$)$ \\
\hline & Ruta et $a l^{\beta 9}$ (USS) & $0.63(0.46$ to 0.78$)$ & $0.57(0.39$ to 0.73$)$ & $1.5(0.89$ to 2.4$)$ & $0.65(0.37$ to 1.1$)$ \\
\hline & Lange et $a{ }^{46}$ (USS) & 0.14 (0.05 to 0.33$)$ & 0.59 (0.41 to 0.74$)$ & $0.33(0.11$ to 1.0$)$ & $1.5(1.0$ to 2.1$)$ \\
\hline & Coari et $a^{\beta 8}$ (USS) & 0.37 (0.15 to 0.66$)$ & 0.50 (0.43 to 0.57$)$ & $0.74(0.35$ to 1.6$)$ & $1.3(0.80$ to 2.0$)$ \\
\hline & Dasgupta et al ${ }^{15}$ (USS) & & & & \\
\hline & Ruta et $a^{\beta 9}$ (USS) & & & & \\
\hline & Lange et $a l^{46}$ (USS) & & & & \\
\hline & Salvarani et a ${ }^{48}$ (MRI) & $0.47(0.25$ to 0.70$)$ & $0.67(0.35$ to 0.88$)$ & $1.4(0.48$ to 4.1$)$ & $0.80(0.41$ to 1.6$)$ \\
\hline \multirow{3}{*}{$\begin{array}{l}\text { Long head biceps tenosynovitis on at least one } \\
\text { side: comparator painful shoulder conditions }\end{array}$} & Coari et $a^{\beta 8}$ (USS) & $0.16(0.069$ to 0.32$)$ & $0.45(0.37$ to 0.54$)$ & $0.28(0.13$ to 0.65$)$ & $1.9(1.5$ to 2.4$)$ \\
\hline & Dasgupta et $a l^{15}$ (USS) & $0.66(0.57$ to 0.74$)$ & 0.60 (0.45 to 0.72$)$ & $1.6(1.1$ to 2.4$)$ & $0.57(0.40$ to 0.80$)$ \\
\hline & Ruta et $a{ }^{\beta 9}$ (USS) & 0.47 (0.35 to 0.59$)$ & 0.80 (0.68 to 0.88$)$ & 2.3 (1.3 to 4.1$)$ & 0.67 (0.51 to 0.87$)$ \\
\hline \multirow[t]{8}{*}{ Long head biceps tenosynovitis on both sides } & Cantini et $a{ }^{\beta 3}$ (USS) & 0.60 (0.47 to 0.72$)$ & $0.96(0.90$ to 0.98$)$ & $15(5.8$ to 41$)$ & $0.42(0.30$ to 0.58$)$ \\
\hline & Frediani et $a{ }^{45}$ (USS) & $0.38(0.26$ to 0.52$)$ & $0.68(0.58$ to 0.76$)$ & $1.2(0.75$ to 1.9$)$ & $0.91(0.71$ to 1.2$)$ \\
\hline & Falsetti et $a{ }^{44}$ (USS) & $0.62(0.44$ to 0.77$)$ & 0.66 (0.48 to 0.80$)$ & 1.8 (1.0 to 3.2$)$ & 0.58 (0.34 to 0.98$)$ \\
\hline & Dasgupta et al ${ }^{15}$ (USS) & $0.37(0.29$ to 0.46$)$ & $0.73(0.66$ to 0.80$)$ & $1.4(0.98$ to 2.0$)$ & $0.86(0.72$ to 1.0$)$ \\
\hline & Cantini et $a^{\beta 3}$ (USS) & 0.47 (0.35 to 0.58$)$ & 0.80 (0.61 to 0.91$)$ & $2.4(0.93$ to 6.0$)$ & 0.67 (0.46 to 0.95$)$ \\
\hline & Frediani et $a \mathrm{f}^{45}$ (USS) & & & & \\
\hline & Falsetti et a $\left.\right|^{44}$ (USS) $^{*}$ & & & & \\
\hline & Dasgupta et al ${ }^{15}$ (USS) & & & & \\
\hline \multirow{2}{*}{$\begin{array}{l}\text { Long head biceps tenosynovitis on both sides: } \\
\text { comparator RA }\end{array}$} & Dasgupta et al ${ }^{15}$ (USS) & 0.37 (0.29 to 0.46$)$ & $0.62(0.48$ to 0.75$)$ & 0.99 (0.63 to 1.5$)$ & $1.0(0.77$ to 1.3$)$ \\
\hline & Ruta et $a{ }^{\beta 9}$ (USS) & 0.30 (0.17 to 0.48$)$ & $0.98(0.86$ to 1.00$)$ & $19(1.2$ to 310$)$ & 0.71 (0.56 to 0.90$)$ \\
\hline Tenosynovitis of hand extensor tendons & Cimmino et $\left.a\right|^{40}$ (MRI) & 0.67 (0.42 to 0.85$)$ & 0.69 (0.42 to 0.87$)$ & $2.2(0.89$ to 5.3$)$ & $0.48(0.22$ to 1.1$)$ \\
\hline \multirow[t]{8}{*}{ Glenohumeral synovitis on at least one side } & Cantini et $a{ }^{\beta 3}$ (USS) & 0.77 (0.65 to 0.86$)$ & $0.42(0.33$ to 0.51$)$ & $1.3(1.1$ to 1.6$)$ & 0.54 (0.32 to 0.92$)$ \\
\hline & Frediani et $a{ }^{45}$ (USS) & $0.66(0.52$ to 0.78$)$ & 0.65 (0.55 to 0.73$)$ & $1.9(1.4$ to 2.6$)$ & 0.52 (0.35 to 0.79$)$ \\
\hline & Falsetti et $a{ }^{44}$ (USS) & $0.66(0.47$ to 0.80$)$ & $0.47(0.31$ to 0.64$)$ & $1.2(0.81$ to 1.9$)$ & $0.74(0.40$ to 1.4$)$ \\
\hline & Dasgupta et al ${ }^{15}$ (USS) & 0.39 (0.30 to 0.48$)$ & 0.71 (0.64 to 0.78$)$ & $1.3(0.96$ to 1.9$)$ & $0.86(0.72$ to 1.0$)$ \\
\hline & Cantini et $a^{\beta 3}$ (USS) & $0.62(0.46$ to 0.76$)$ & 0.58 (0.45 to 0.69$)$ & $1.5(1.2$ to 1.7$)$ & $0.66(0.50$ to 9.9$)$ \\
\hline & Frediani et $a{ }^{45}$ (USS) & & & & \\
\hline & Falsetti et a ${ }^{44}$ (USS) & & & & \\
\hline & Dasgupta et al ${ }^{15}$ (USS) & & & & \\
\hline \multirow{4}{*}{$\begin{array}{l}\text { Glenohumeral synovitis on at least one side: } \\
\text { comparator RA }\end{array}$} & Lange et $a f^{46}$ (USS) & $0.41(0.23$ to 0.61$)$ & $0.34(0.20$ to 0.53$)$ & $0.62(0.35$ to 1.1$)$ & 1.7 (0.93 to 3.2$)$ \\
\hline & Coari et $a^{\beta 8}$ (USS) & $0.66(0.48$ to 0.80$)$ & $0.52(0.42$ to 0.62$)$ & $1.4(0.99$ to 1.9$)$ & $0.66(0.39$ to 1.1$)$ \\
\hline & Ruta et $a^{\beta 9}$ (USS) & $0.20(0.10$ to 0.37$)$ & 0.57 (0.39 to 0.73$)$ & $0.46(0.20$ o 1.1$)$ & 1.4 (0.99 to 2.0$)$ \\
\hline & Dasgupta et $a l^{15}$ (USS) & 0.39 (0.30 to 0.48$)$ & $0.63(0.49$ to 0.75$)$ & $1.1(0.67$ to 1.6$)$ & $0.97(0.75$ to 1.3$)$ \\
\hline
\end{tabular}




\section{Anatomical finding}

Studies (imaging modality)

Sensitivity

(95\% Cl), \%

Specificity

(95\% Cl), \%

Positive likelihood

Lange et $a f^{46}$ (USS)†

Coari et $a^{\beta 8}$ (USS)†

$0.41(0.26$ to 0.58$)$

0.53 (0.45 to 0.62$) \quad 0.88$ (0.58 to 1.3$)$ ratio $(95 \% \mathrm{Cl})$

Ruta et $a{ }^{\beta 9}$ (USS)

Dasgupta et al ${ }^{15}$ (USS)

Glenohumeral synovitis on at least one side:

comparator painful shoulder conditions

Glenohumeral synovitis on both sides: comparator Dasgupta et al ${ }^{15}$

RA

Hip synovitis on at least one side

Hip synovitis on both sides

Shoulder region uptake

Bilateral shoulder region inflammation

Bilateral shoulder region inflammation: comparator Dasgupta et al ${ }^{15}$ (USS)

painful shoulder conditions

Bilateral shoulder region inflammation: comparator Dasgupta et al ${ }^{15}$ (USS)

RA

Hip region inflammation: comparator $\mathrm{RA}$

Salvarani et al ${ }^{48}(\mathrm{MRI})$

Coari et $a^{\beta 8}$ (USS)

Dasgupta et al ${ }^{15}$ (USS)

Ruta et $a l^{39}$ (USS)

Dasgupta et $a 1^{15}$

Falsetti et $a{ }^{44}$

Frediani et $a{ }^{45}$

Ruta et a ${ }^{\beta 9}$

Cantini et $a^{\beta 4}$ (USS)

Frediani et $a f^{45}$ (USS)

Falsetti et $a{ }^{14}$ (USS)

Dasgupta et al ${ }^{15}$ (USS)

Cantini et $a^{\beta 4}$ (USS)

Frediani et $a f^{45}$ (USS)

Falsetti et $a{ }^{44}$ (USS)

Dasgupta et al ${ }^{15}$ (USS)

Cantini et $a{ }^{\beta 4}$ (MRI)

Yamashita et $a^{35}$ (PET/CT)

Dasgupta et al ${ }^{15}$ (USS)

Frediani et a ${ }^{45}$ (USS)

Yamashita et $a^{\beta 5}$ (PET/CT)

Dasgupta et al ${ }^{15}$ (USS)

Macchioni et $a^{R^{3}}$ (USS)

Macchioni 2013 (USS

Macchioni 2013 (USS
Dasgupta et al ${ }^{15}$ (USS)

Dasgupta et al ${ }^{15}$ (USS)

0.77 (0.50 to 0.92$)$

0.66 (0.48 to 080$)$

0.39 (0.30 to 0.48$)$

$0.12(0.058$ to 0.22$)$

$0.26(0.19$ to 0.35$)$

0.48 (0.31 to 0.66$)$

0.52 (0.39 to 0.65$)$

0.26 (0.19 to 0.35$)$

0.03 (0.059 to 0.17$)$

0.45 (0.25 to 0.66$)$

0.40 (0.28 to 0.54$)$

0.24 (0.12 to 0.42 )

0.26 (0.19 to 0.36 )

0.33 (0.24 to 0.43 )

$0.44(0.19$ to 0.73$)$

0.77 (0.69 to 0.84 )

0.76 (0.62 to 0.86$)$

0.93 (0.84 to 0.97 )

0.83 (0.76 to 0.88 )

0.66 (0.48 to 0.80$)$

0.78 (0.69 to 0.85 )

0.70 (0.55 to 0.81$)$

0.90 (0.74 to 0.97 )

0.55 (0.40 to 0.69 )

0.81 (0.72 to 0.87$)$

0.88 (0.72 to 0.95$)$

0.81 (0.73 to 0.87$)$

0.78 (0.66 to 0.87 )

$1.4(0.72$ to 2.7$)$ 2.9 (1.9 to 4.3 )

$1.6(0.92$ to 2.8$)$

1.8 (0.54 to 5.7$)$

1.5 (0.97 to 2.4$)$

$1.4(0.76$ to 2.6$)$

2.4 (1.50 to 3.7 )

0.86 (0.50 to 1.5$)$

$0.33(0.037$ to 3.0$)$

1.0 (0.55 to 1.8$)$

2.1 (1.2 to 3.6$)$

1.9 (0.63 to 5.9$)$

1.4 (0.87 to 2.3$)$

1.5 (1.0 to 2.2 )

0.85 (0.64 to 0.95$)$

0.86 (0.60 to 0.96$)$

0.18 (0.12 to 0.26$)$

0.32 (0.21 to 0.46$)$

0.86 (0.60 to 0.96$)$

0.59 (0.50 to 0.68 )

0.45 (0.39 to 0.51 )

0.59 (0.50 to 0.68 )

0.50 (0.24 to 0.76$)$

0.65 (0.41 to 0.83 )

0.92 (0.85 to 0.95$)$

0.83 (0.75 to 0.89$)$

0.29 (0.13 to 0.53$)$

0.57 (0.49 to 0.65$)$

0.60 (0.47 to 0.72$)$

0.74 (0.61 to 0.85 )

1.7 (0.89 to 3.2$)$ 2.4 (1.2 to 4.8$)$

2.1 (1.0 to 4.3$)$

1.88 (1.0 to 3.4$)$

$1.2(0.84$ to 1.8$)$

1.4 (1.1 to 1.7$)$

$1.1(0.79$ to 1.6$)$

2.3 (1.4 to 3.9$)$

$0.59(0.50$ to 0.68$) \quad 0.35(0.23$ to 0.49$) \quad 0.91(0.70$ to 1.2$)$

$0.56(0.49$ to 0.63$) \quad 0.74(0.60$ to 0.85$) \quad 2.2(1.3$ to 3.7$)$

0.38 (0.30 to 0.47$) \quad 0.70(0.55$ to 0.81$) \quad 1.3(0.77$ to 2.1$)$

0.38 (0.30 to 0.47$) \quad 0.83(0.70$ to 0.91$) \quad 2.3(1.2$ to 4.4$)$

0.33 (0.26 to 0.42$) \quad 0.84(0.77$ to 0.89$) \quad 2.1(1.3$ to 3.2$)$

0.34 (0.27 to 0.41$) \quad 0.77$ (0.68 to 0.85$) \quad 1.5(0.97$ to 2.3$)$

Dasgupta et $a l^{15}$ (USS)

Macchioni et $a R^{3}$ (USS)

Note these are reported to 2 significant figures but note Cls are often wide. Sensitivity, specificity, positive likelihood ratio and negative likelihood ratio are given for individual tests or, where possible, a summary value is calculated by meta-analysis. Sensitivity and specificity are given to two decimal places. Likelihood ratios are given to two significant figures unless $<0.1$ or $>10$. ${ }^{*}$ Outlier by visual inspection of HsROC.

tInfluential outlier (Cooks' distance $>3$ ). "Shoulder region inflammation", "hip region inflammation" are defined as per ACR/EULAR provisional classification criteria for PMR. ${ }^{15}$

ACR, American College of Rheumatology; EULAR, the European League Against Rheumatism; HsROC, Hierarchial Summary Receiver-Operator Characteristic Curve; PMR, polymyalgia

rheumatica.

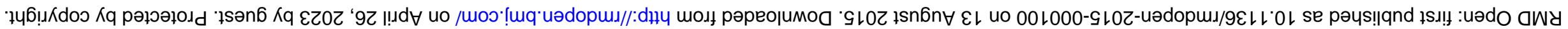




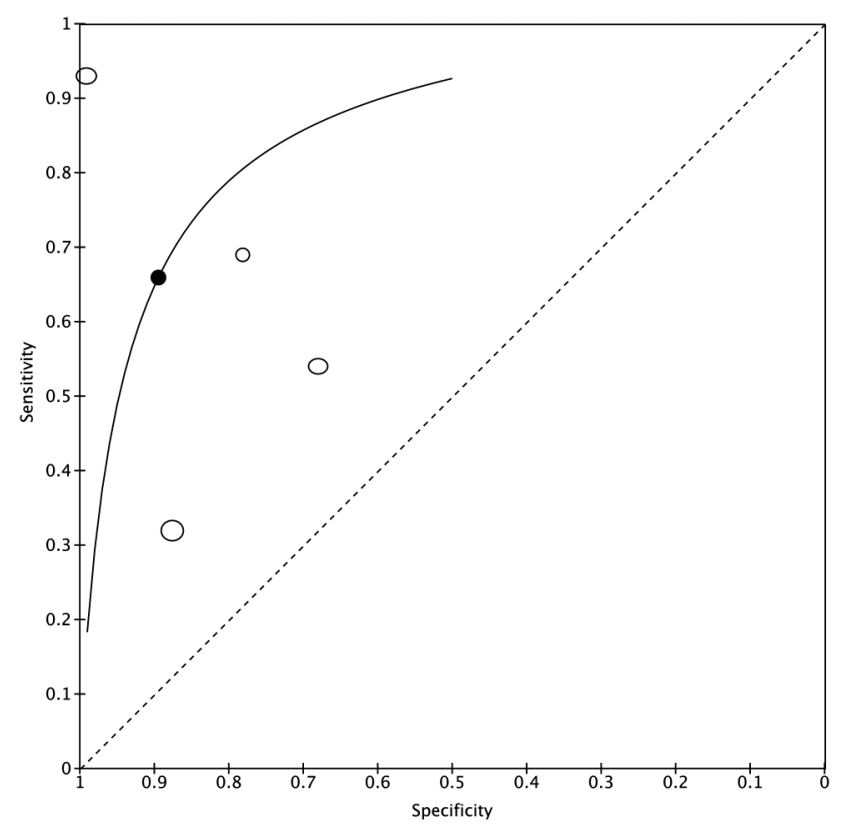

Figure 2 Summary ROC plot for bilateral subacromial bursitis. ROC, receiver operating characteristic.

combined. First, different anatomical sites from each region were combined: shoulder region inflammation was defined as SAB, fluid around the long head of biceps tendon, OR glenohumeral synovitis; hip region inflammation was defined as coxofemoral synovitis OR trochanteric bursitis. ${ }^{15}$ This use of OR had the effect of increasing sensitivity of the criteria. Second, based on the regression modelling used to define the final classification criteria set, one point was allocated for bilateral shoulder region inflammation, and one point for shoulder region inflammation plus hip region inflammation. This requirement for two regions involved had the effect of increasing specificity of the criteria. Bilateral shoulder region involvement had a sensitivity of $59 \%$ (50\% to $68 \%$ ) and specificity $57 \%$ ( $49 \%$ to $65 \%$ ), whereas having one shoulder and one hip involved had a sensitivity $33 \%$ (26\% to $42 \%)$ and specificity $84 \%(77 \%$ to $89 \%) .{ }^{15}$ This may reflect the requirement for shoulder symptoms for inclusion of both patients and controls, whereas PMR characteristically causes symptoms at shoulders as well as hips. A later study produced similar sensitivity/specificity data, with the caveat that its control population was patients with early RA, and the completeness of sonographer and diagnostician blinding to each other's findings was unclear. ${ }^{23}$

\section{Change with treatment}

Comparison of before-treatment and after-treatment findings was reported for musculoskeletal USS, ${ }^{28-30}$ MRI $^{31}$ and FDG-PET. ${ }^{41}$ After 4 weeks of glucocorticoid treatment, shoulder USS normalised in half of the patients who had had bilateral USS abnormalities before treatment, and this persisted to 6 months. ${ }^{28}$ In a second study, 11/24 patients had power Doppler signal (indicating microvascular hyperaemia, and suggesting chronicity of inflammation) in at least one shoulder structure; this was present in only $1 / 24$ patients at 6 months. PMR still had abnormalities in shoulder ultrasound at 6 months compared to a group of 21 'normal' patients, but this was not seen for hip ultrasound findings. ${ }^{30}$

\section{Prognosis}

In 57 patients with PMR, the presence of power Doppler signal prior to treatment in articular/periarticular shoulder structures significantly predicted PMR relapse/recurrence after 6 months. ${ }^{29}$

\section{Direct comparison of test accuracy using paired data}

Only two couples of tests had sufficient paired data $(\geq 4$ studies) for our analysis. The paired comparisons were only made where the relevant tests were carried out on cases and controls in all studies, so the same cases and controls had both tests. Ultrasound detection of bilateral $\mathrm{SAB}$ was compared to ultrasound detection of hip synovitis, but the two sROC's had different shapes and comparison of overall accuracy was not possible. Ultrasound detection of subacaromial-subdeltoid bursitis was compared to ultrasound detection of glenohumeral synovitis. The two sROC's had similar shapes, and we found ultrasound detection of SAB to be significantly more accurate than ultrasound detection of glenohumeral synovitis, for the diagnosis of PMR $(\mathrm{p}=0.004)$.

\section{DISCUSSION}

Our objective was to determine whether musculoskeletal imaging is accurate enough to be useful to support clinical diagnosis of PMR. Although MRI and PET/CT revealed potentially characteristic features of focal inflammation between vertebral processes and within the pelvis, only the USS studies had enough control patients with inflammatory diseases for precise estimates of diagnostic accuracy. The most informative single USS feature appeared to be bilateral $\mathrm{SAB}$, with a specificity of $89 \%$ (95\% CI $66 \%$ to $97 \%$ ) and sensitivity $66 \%$ (43\% to $87 \%$ ); however, the earliest study reported much higher diagnostic accuracy than subsequent studies. In general, substantial clinical and statistical between-study heterogeneity was noted, including important biases, and therefore the absolute sensitivity/specificity estimates given here must be interpreted with great caution. The effects of between-study heterogeneity can be minimised where each study reported the same two tests; this pairwise comparison across four studies showed that $\mathrm{SAB}$ was significantly more accurate than glenohumeral synovitis for PMR diagnosis. This suggests that it might not be appropriate to weight these two features equally in diagnosing PMR, as has been suggested by the latest criteria set. ${ }^{15}$

Several potential biases were identified during the quality assessment. First, all studies, except one, had a case-control study design. This would introduce 
spectrum bias and produce heterogeneity in the specificity estimates depending on how the controls were recruited. The only study with a diagnostic cohort design suffered from incorporation bias because the ultrasound was used to help make the diagnosis. Second, most of the reports contained little detail on how blinding was achieved and maintained. This is particularly difficult for USS, which requires close patient contact. Recruitment of two controls following each case could have compromised blinding and was associated with much higher estimates of diagnostic accuracy. ${ }^{33} 34$ Blinding of the treating rheumatologist and the patient until after the final adjudication of reference-standard clinical diagnosis (which may be 1 year later), would require explicit patient consent and may not always have been possible. There was often insufficient detail on whether and how the patients themselves were blinded to their imaging findings, and on how frequently the treating clinician had to be unblinded or patients excluded from analysis because of unexpected findings on the scan (particularly relevant for MRI and PET studies). Lastly, intra/inter-rater reliability of imaging test was rarely fully reported, although this was arguably unlikely to introduce a systematic bias.

Some limitations of this analysis could have made imaging appear less accurate than it really is. First, the use of binary scores (present/absent) rather than grades of intensity of inflammation or number of sites involved is a limitation of diagnostic accuracy meta-analysis methods. Second, the necessity of using rheumatologist diagnosis as an (imperfect) reference standard; for future studies, adding a 'test of treatment ${ }^{42}$ might be used to improve the reference standard, since ultrasound abnormalities were associated with complete response to glucocorticoid therapy. ${ }^{30}$ Third, it is not known whether adding power Doppler to the ultrasound might offer superior diagnostic accuracy for PMR compared to grey-scale ultrasound alone.

Overall, the accuracy of musculoskeletal imaging tests cannot currently be accurately quantified for clinical diagnosis of PMR, primarily due to the limited amount of published data and biases in the studies. The reference standard is still rheumatologist diagnosis, which may use clinical intuition rather than formal criteria ${ }^{43}$ we might expect that tests adding additional information, including imaging tests, might help in 'grey cases' where the clinical diagnosis is not clear-cut, but there are no studies recruiting these 'grey cases' and evaluating them without incorporation bias. Finally, if the prognostic value of imaging were known, this might also have value for clinical practice and perhaps even for patient classification. This type of evidence would help determine the optimal place of imaging tests in diagnostic care pathways for patients with suspected PMR.

\footnotetext{
Author affiliations

${ }^{1}$ Leeds Institute of Rheumatic and Musculoskeletal Medicine, University of Leeds, Leeds, UK and NIHR Leeds Musculoskeletal Biomedical Research Unit, UK
}

${ }^{2}$ Leeds Teaching Hospitals NHS Trust, Leeds, UK

${ }^{3}$ Harrogate and District NHS Foundation Trust, Harrogate, UK

${ }^{4}$ York Teaching Hospital NHS Foundation Trust, UK

${ }^{5}$ University of Adelaide, The Queen Elizabeth Hospital, Adelaide, Australia

${ }^{6}$ Department of Health Services Research and Policy, London School of

Hygiene and Tropical Medicine, London, UK

${ }^{7}$ University of Sydney, Sydney, Australia

${ }^{8}$ Huntington Disease Service, Westmead Hospital, Sydney, Australia

${ }^{9}$ Southend University Hospitals NHS Trust, UK

${ }^{10}$ Leeds Institute of Health Sciences, University of Leeds, UK

Acknowledgements The authors thank the individuals who kindly provided more detailed data or additional information from their original published studies for the purpose of this analysis: Cynthia Crowson, BS, MS, Mayo Clinic, USA; Eric Matteson, BA, MD, MPH, Mayo Clinic, USA; Santiago Ruta, MD, Hospital Italiano de Buenos Aires, Argentina; Dario Camellino, MD, and Marco Cimmino, MD, Università di Genova, Italy; Paolo Falsetti MD, PhD, San Donato Hospital, Arezzo, Italy; and Hiroyuki Takahashi MD, National Center for Global Health and Medicine, Shinjuku-ku, Tokyo, Japan. All those named above have given their permission to be included in the acknowledgements.

Contributors SLM wrote the review protocol including search strategies and clinical perspective, retrieved papers, screened papers for inclusion, appraised quality, extracted and managed data, contacted authors, performed the analysis and wrote the review; GK helped design the review including clinical perspective, screened papers for inclusion, appraised quality, extracted data and co-wrote the review; CLH helped design the review, retrieved papers, appraised quality and extracted data; BD conceived the review, contacted authors and provided clinical perspective; RJW provided clinical perspective; JCW helped design the review, and provided methodological support and general advice; CL performed the analysis and provided methodological support; AH provided methodological support. All the authors contributed to design and/or interpretation, revised the manuscript for important intellectual content and approved the final version.

Funding This report is original research arising from a Clinician Scientist Fellowship Award to Dr Sarah Mackie, supported by the National Institute for Health Research. The views expressed in this publication are those of the authors and not necessarily those of the National Health Service, the National Institute for Health Research or the UK Department of Health.

Competing interests SLM is funded by a NIHR Clinician Scientist Award and is currently conducting a diagnostic accuracy study of ultrasound in the diagnosis of PMR, ADDRESS-PMR. GK has been trained in musculoskeletal ultrasound. RJW is a musculoskeletal ultrasound trainer and clinical researcher. JCW is fully funded by the University of Leeds and has no potential competing interests to declare. BD is a member of Advisory Boards on Napp and Mundipharma for trial design. SLM, AH, RJW and BD are currently revising the British Society for Rheumatology clinical practice guidelines on diagnosis and treatment of PMR.

Provenance and peer review Not commissioned; externally peer reviewed.

Data sharing statement Data used in the preparation of this review are available on request from the corresponding author. SLM had full access to all the data in the study and takes responsibility for the integrity of the data and the accuracy of the data analysis.

Open Access This is an Open Access article distributed in accordance with the terms of the Creative Commons Attribution (CC BY 4.0) license, which permits others to distribute, remix, adapt and build upon this work, for commercial use, provided the original work is properly cited. See: http:// creativecommons.org/licenses/by/4.0/

\section{REFERENCES}

1. Crowson CS, Matteson EL, Myasoedova E, et al. The lifetime risk of adult-onset rheumatoid arthritis and other inflammatory autoimmune rheumatic diseases. Arthritis Rheum 2011;63:633-9.

2. Lawrence RC, Felson DT, Helmick CG, et al. Estimates of the prevalence of arthritis and other rheumatic conditions in the United States. Part II. Arthritis Rheum 2008;58:26-35. 
3. Mackie SL, Mallen CD. Polymyalgia rheumatica. BMJ 2013;347: f6937.

4. Hutchings A, Hollywood J, Lamping DL, et al. Clinical outcomes, quality of life, and diagnostic uncertainty in the first year of polymyalgia rheumatica. Arthritis Rheum 2007;57:803-9.

5. Mazzantini M, Torre C, Miccoli M, et al. Adverse events during longterm low-dose glucocorticoid treatment of polymyalgia rheumatica: a retrospective study. J Rheumatol 2012;39:552-7.

6. Hancock AT, Mallen CD, Muller S, et al. Risk of vascular events in patients with polymyalgia rheumatica. CMAJ 2014;28:140266.

7. Wood-Baker R, Walters J, Walters EH. Systemic corticosteroids in chronic obstructive pulmonary disease: an overview of Cochrane systematic reviews. Respir Med Mar 2007;101:371-7.

8. Wei L, MacDonald TM, Walker BR. Taking glucocorticoids by prescription is associated with subsequent cardiovascular disease. Ann Intern Med 2004;141:764-70.

9. Dasgupta B, Borg FA, Hassan N, et al. BSR and BHPR guidelines for the management of polymyalgia rheumatica. Rheumatology (Oxford) 2010;49:186-90.

10. Camellino D, Cimmino MA. Imaging of polymyalgia rheumatica: indications on its pathogenesis, diagnosis and prognosis. Rheumatology (Oxford) 2012;51:77-86.

11. McGonagle $\mathrm{D}$, Pease $\mathrm{C}$, Marzo-Ortega $\mathrm{H}$, et al. The case for classification of polymyalgia rheumatica and remitting seronegative symmetrical synovitis with pitting edema as primarily capsular/ entheseal based pathologies. J Rheumatol 2000;27:837-40.

12. McGonagle D, Pease C, Marzo-Ortega $\mathrm{H}$, et al. Comparison of extracapsular changes by magnetic resonance imaging in patients with rheumatoid arthritis and polymyalgia rheumatica. J Rheumatol 2001;28:1837-41.

13. Marzo-Ortega $\mathrm{H}$, Rhodes LA, Tan AL, et al. Evidence for a different anatomic basis for joint disease localization in polymyalgia rheumatica in comparison with rheumatoid arthritis. Arthritis Rheum 2007:56:3496-501.

14. Cimmino MA, Camellino D, Paparo F, et al. High frequency of capsular knee involvement in polymyalgia rheumatica/giant cell arteritis patients studied by positron emission tomography. Rheumatology (Oxford) 2013;52:1865-72.

15. Dasgupta B, Cimmino MA, Maradit-Kremers H, et al. 2012 provisiona classification criteria for polymyalgia rheumatica: a European League Against Rheumatism/American College of Rheumatology collaborative initiative. Ann Rheum Dis 2012;71:484-92.

16. Blankenbaker DG, Ullrick SR, Davis KW, et al. Correlation of MRI findings with clinical findings of trochanteric pain syndrome. Skeletal Radiol 2008;37:903-9.

17. lagnocco A, Filippucci E, Riente L, et al. Ultrasound imaging for the rheumatologist XLI. Sonographic assessment of the hip in OA patients. Clin Exp Rheumatol 2012;30:652-7.

18. Whiting PF, Rutjes AW, Westwood ME, et al. QUADAS-2: a revised tool for the quality assessment of diagnostic accuracy studies. Ann Intern Med 2011;155:529-36.

19. Harbord RM, Whiting PF. Metandi: meta-analysis of diagnostic accuracy using hierarchial logistic regression. Stata $J$ 2009;9:211-29.

20. Herbert R. Confidence Interval Calculator. 2013. http://www.pedro. org.au/english/downloads/confidence-interval-calculator/ (accessed 16 Apr 2014).

21. Rutter CM, Gatsonis CA. A hierarchical regression approach to meta-analysis of diagnostic test accuracy evaluations. Stat Med 2001;20:2865-84.

22. Macaskill P, Gatsonis CA, Deeks JJ, et al. Chapter 10: analysing and presenting results. In: Collaboration TC, ed. Cochrane handbook for systematic reviews of diagnostic test accuracy. 2010.

23. Macchioni $\mathrm{P}$, Boiardi L, Catanoso M, et al. Performance of the new 2012 EULAR/ACR classification criteria for polymyalgia rheumatica: comparison with the previous criteria in a single-centre study. Ann Rheum Dis 2014;73:1190-3.

24. Takahashi $\mathrm{H}$, Yamashita $\mathrm{H}$, Kubota $\mathrm{K}$, et al. Differences in FDG PET/CT between EORA and PMR. Mod Rheumatol 2014:1-6.

25. Camellino D, Paparo F, Morbelli S, et al. Interspinous bursitis is common in polymyalgia rheumatica, but is not associated with spinal pain. Arthritis Res Ther 2014;16:492.
26. Pego-Reigosa JM, Rodriguez-Rodriguez M, Hurtado-Hernandez Z, et al. Calcium pyrophosphate deposition disease mimicking polymyalgia rheumatica: a prospective followup study of predictive factors for this condition in patients presenting with polymyalgia symptoms. Arthritis Rheum 2005;53:931-8.

27. O'Duffy JD, Wahner HW, Hunder GG. Joint imaging in polymyalgia rheumatica. Mayo Clin Proc 1976;51:519-24.

28. Jimenez-Palop M, Naredo E, Humbrado L, et al. Ultrasonographic monitoring of response to therapy in polymyalgia rheumatica. Ann Rheum Dis 2010;69:879-82.

29. Macchioni P, Catanoso MG, Pipitone N, et al. Longitudinal examination with shoulder ultrasound of patients with polymyalgia rheumatica. Rheumatology (Oxford) 2009;48:1566-9.

30. Matteson EL, Maradit-Kremers H, Cimmino MA, et al. Patient-reported outcomes in polymyalgia rheumatica. J Rheumatol 2012;39:795-803

31. Salvarani C, Cantini F, Olivieri I, et al. Corticosteroid injections in polymyalgia rheumatica: a double-blind, prospective, randomized, placebo controlled study. J Rheumatol 2000;27:1470-6.

32. Lijmer JG, Mol BW, Heisterkamp S, et al. Empirical evidence of design-related bias in studies of diagnostic tests. JAMA 1999;282:1061-6.

33. Cantini F, Salvarani C, Olivieri I, et al. Shoulder ultrasonography in the diagnosis of polymyalgia rheumatica: a case-control study. $J$ Rheumatol 2001;28:1049-55.

34. Cantini F, Niccoli L, Nannini C, et al. Inflammatory changes of hip synovial structures in polymyalgia rheumatica. Clin Exp Rheumatol 2005;23:462-8.

35. Yamashita H, Kubota K, Takahashi Y, et al. Whole-body fluorodeoxyglucose positron emission tomography/computed tomography in patients with active polymyalgia rheumatica: evidence for distinctive bursitis and large-vessel vasculitis. Mod Rheumatol 2012;22:705-11.

36. Salvarani $C$, Barozzi L, Cantini $F$, et al. Cervical interspinous bursitis in active polymyalgia rheumatica. Ann Rheum Dis 2008;67:758-61.

37. Salvarani C, Barozzi L, Boiardi L, et al. Lumbar interspinous bursitis in active polymyalgia rheumatica. Clin Exp Rheumatol 2013;31:526-31.

38. Coari G, Paoletti F, lagnocco A. Shoulder involvement in rheumatic diseases. Sonographic findings. J Rheumatol 1999;26:668-73.

39. Ruta S, Rosa J, Navarta DA, et al. Ultrasound assessment of new onset bilateral painful shoulder in patients with polymyalgia rheumatica and rheumatoid arthritis. Clin Rheumatol 2012;31:1383-7.

40. Cimmino MA, Parodi M, Zampogna G, et al. Polymyalgia rheumatica is associated with extensor tendon tenosynovitis but not with synovitis of the hands: a magnetic resonance imaging study. Rheumatology (Oxford) 2011;50:494-9.

41. Blockmans D, De Ceuninck L, Vanderschueren S, et al. Repetitive 18-fluorodeoxyglucose positron emission tomography in isolated polymyalgia rheumatica: a prospective study in 35 patients. Rheumatology (Oxford) 2007;46:672-7.

42. Glasziou $\mathrm{P}$, Rose $\mathrm{P}$, Heneghan $\mathrm{C}$, et al. Diagnosis using "test of treatment". BMJ 2009;338:b1312.

43. Spiera R, Westhovens R. Provisional diagnostic criteria for polymyalgia rheumatica: moving beyond clinical intuition? Ann Rheum Dis 2012;71:475-6.

44. Falsetti $\mathrm{P}, \mathrm{Acciai} \mathrm{C}$, Volpe $\mathrm{A}$, et al. Ultrasonography in early assessment of elderly patients with polymyalgic symptoms: a role in predicting diagnostic outcome? Scand J Rheumatol 2011;40:57-63.

45. Frediani B, Falsetti $\mathrm{P}$, Storri $\mathrm{L}$, et al. Evidence for synovitis in active polymyalgia rheumatica: sonographic study in a large series of patients. J Rheumatol 2002;29:123-30.

46. Lange U, Piegsa M, Teichmann J, et al. Ultrasonography of the glenohumeral joints - a helpful instrument in differentiation in elderly onset rheumatoid arthritis and polymyalgia rheumatica. Rheumatol Int 2000;19:185-9.

47. Lange U, Teichmann J, Stracke $\mathrm{H}$, et al. Elderly onset rheumatoid arthritis and polymyalgia rheumatica: ultrasonographic study of the glenohumeral joints. Rheumatol Int 1998;17:229-32.

48. Salvarani C, Cantini F, Olivieri I, et al. Proximal bursitis in active polymyalgia rheumatica. Ann Intern Med 1997;127:27-31. 